\title{
Neuronal Responses in the Nucleus Accumbens Shell during Sexual Behavior in Male Rats
}

\author{
Jumpei Matsumoto, ${ }^{1}$ Susumu Urakawa, ${ }^{2}$ Etsuro Hori, ${ }^{1}$ Mariana F. P. de Araujo, ${ }^{1}$ Yasuo Sakuma, ${ }^{3}$ Taketoshi Ono, ${ }^{2}$ \\ and Hisao Nishijo ${ }^{1}$ \\ ${ }^{1}$ System Emotional Science and 2Department of Judo Neurophysiotherapy, Graduate School of Medicine and Pharmaceutical Sciences, University of \\ Toyama, Toyama 930-0194, Japan, and 32Department of Physiology, Nippon Medical School, Bunkyo-ku, Tokyo 113-8602, Japan
}

\begin{abstract}
Previous behavioral studies have indicated that the nucleus accumbens (NAc) shell of a male rat is involved in its sexual behavior; however, no previous studies have investigated neuronal activities in the male rat NAc shell during sexual behavior. To investigate this issue, we recorded single unit activities in the NAc shell of male rats during sexual behavior. Of 123 NAc shell neurons studied, 53, 47, and 40 neurons exhibited significantly changed firing rates at various times during intromission, genital auto-grooming, and sniffing of females, respectively. The two types of NAc shell neurons [putative fast spiking interneurons (pFSIs) and medium spiny neurons (pMSNs)] responded differently during sexual behavior. First, more pFSIs than pMSNs exhibited inhibitory responses to thrusting with intromission and genital grooming, while pFSIs and pMSNs responded similarly to sniffing of females. Second, both pFSIs and pMSNs responded differently to thrusting with and without intromission. Furthermore, NAc shell neuronal activity was significantly different across the different phases of sexual behavior, and the number of NAc shell neurons with delta oscillation, which is related to behavioral inhibition, and high gamma oscillation, which is related to reward perception, increased after ejaculation. Together, our results suggest that the NAc shell is deeply involved in sexual behavior, and changes in NAc shell neuronal activity are related to performance of sexual behavior, encoding cues or contexts related to sexual behavior, reward-related processing, and the inhibition of sexual behavior after ejaculation.
\end{abstract}

\section{Introduction}

Sexual behavior in male rats consists of two major types, precopulatory and copulatory. In precopulatory behavior, male rats chase females and sniff female odors to stimulate sexual excitation in itself and its partner. Copulatory behavior is categorized into three main groups: mounting (the male mounts a sexually receptive female), intromission (after mounting, the penis becomes erect and the male inserts it into the vagina during thrusts), and ejaculation (after a series of mounts and intromissions, the male ejaculates). Following ejaculation, the male does not engage in sexual activity with the female for some period of time (postejaculatory interval).

The nucleus accumbens (NAc) is one of the regions in the brain that is involved in sexual behavior of male rats. Previous lesion and pharmacological studies reported that various manipulations in the NAc affected latency to copulation, but not performance of copulation itself (Hull et al., 1986; Liu et al., 1998).

Received Oct. 11, 2011; revised Dec. 4, 2011; accepted Dec. 19, 2011.

Author contributions: J.M., S.U., Y.S., and H.N. designed research; J.M., S.U., E.H., and M.F.P.d.A. performed research; Y.S. and T.O. contributed unpublished reagents/analytic tools; J.M., M.F.P.d.A., Y.S., T.O., and H.N. analyzed data; J.M. and H.N. wrote the paper.

This research was supported in part by the JSPS Asian Core Program, and a Grant-in-Aid for Scientific Research (A) (22240051).

Correspondence should be addressed to Dr. Hisao Nishijo, System Emotional Science, Graduate School of Medicine and Pharmaceutical Sciences, University of Toyama, Sugitani 2630, Toyama 930-0194, Japan. E-mail: nishijo@med.u-toyama.ac.jp.

DOI:10.1523/JNEUROSCI.5140-11.2012

Copyright $\odot 2012$ the authors $\quad 0270-6474 / 12 / 321672-15 \$ 15.00 / 0$
Moreover, that interaction between the amygdala and NAc is important for instrumental responses to conditioned sexual reinforcers (Everitt et al., 1989). These findings suggest that the NAc plays an important role in appetitive behaviors for sexual reward, but not in copulation performance (Everitt, 1990). Consistent with this idea, previous studies using natural rewards rather than sexual rewards suggest that the NAc is involved in reward-related processing (Wise, 2004) and associative learning that links cues or contexts with rewards according to inputs from the amygdala or hippocampus (Cador et al., 1989; Everitt et al., 1991; Ito et al., 2008).

On the other hand, a few studies have demonstrated that manipulation of the NAc affected copulation performance (Fernández-Guasti et al., 1992; Kippin et al., 2004). The NAc plays a critical role in partner-preference formation in male prairie voles (Aragona et al., 2006); moreover, in male rats, it is activated by odors associated with females (West et al., 1992; Kippin et al., 2003). Partner preference and attraction to female odors evolves through experience of copulation with females (Pfaus et al., 2001). Together, these previous studies suggest that the NAc may encode rewards, cues, and/or contexts related to sexual behavior.

Anatomically, the NAc consists of the shell and core. The former is related more closely to innate, unconditioned behaviors when compared with the latter (Voorn et al., 2004), and it plays a critical role in partner-preference formation in male prairie voles (Aragona et al., 2006). Furthermore, the NAc shell (NAcS) receives inputs from the medial preoptic area (MPOA) and medial 


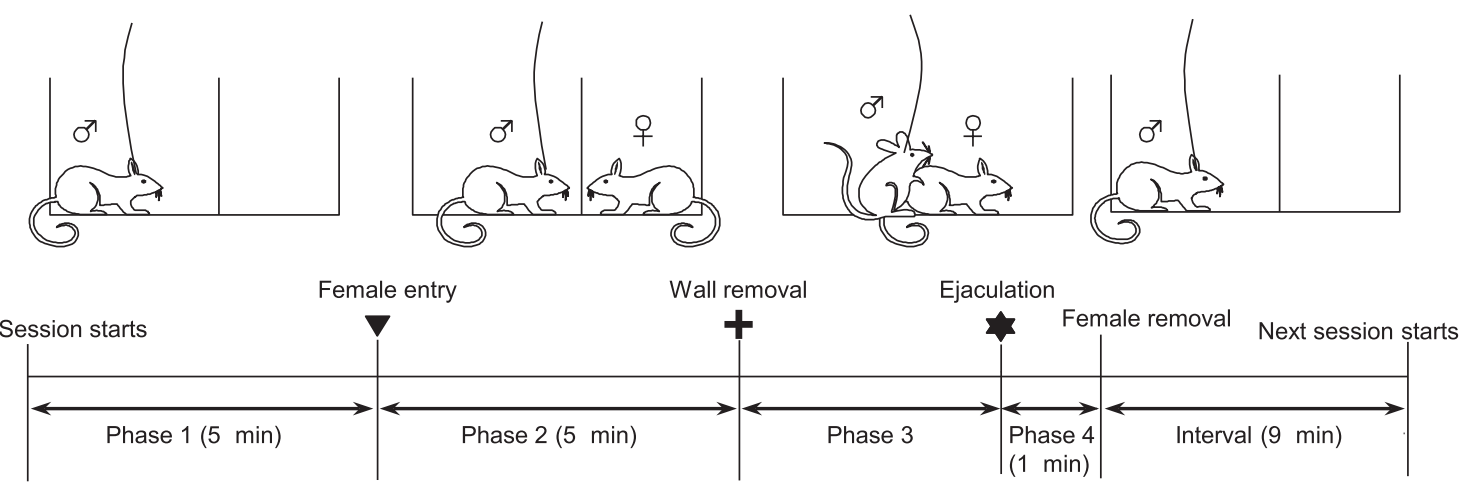

Figure 1. Recording session schedule and definition of the four phases. The experiment was conducted sequentially from Phase 1 to Phase 4. Phase 1: a male rat was placed alone in one of the two compartments of a recording chamber for $5 \mathrm{~min}$. Phase 2: a female was placed in the other compartment for $5 \mathrm{~min}$, but the male could not access the female because of a double mesh wall. Phase 3: the wall was removed, and the male could freely interact with the female until the male ejaculated. Phase 4: 1 min after ejaculation. The session comprising the four phases was repeated three times for each recording experiment.

\section{A Copulatory behaviors}

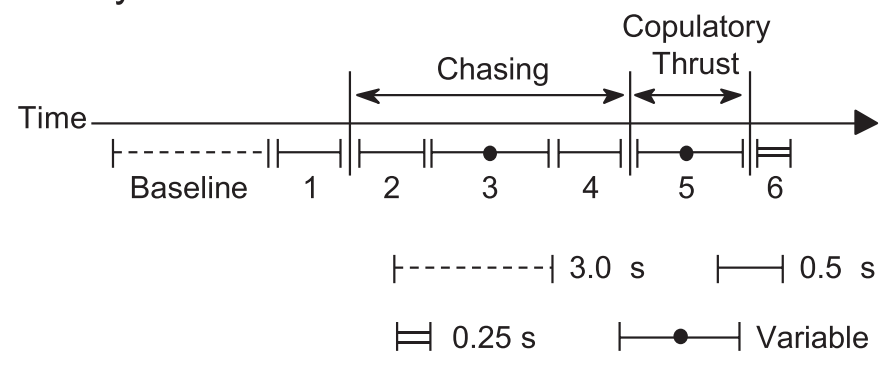

\section{B Genital grooming}

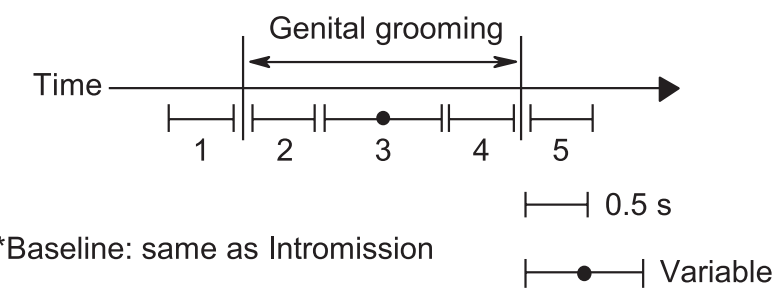

\section{Sniffing to an inaccessible female}

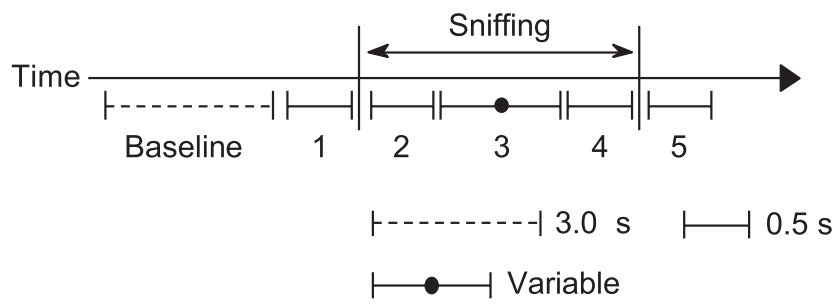

Figure 2. Definition of the behavioral periods used for statistical analysis of neuronal responses to sexual behavior. $A$, Definition of the periods for the three copulatory behaviors (mount + thrust, intromission + thrust, ejaculation + intromission + thrust). A baseline period was defined as the $3.0 \mathrm{~s}$ period from $-3.5 \mathrm{~s}$ to $-0.5 \mathrm{~s}$ before onset of chasing. Periods 1 and 2 were defined as the $0.5 \mathrm{~s}$ periods before and after onset of chasing, respectively. Period 4 was defined as the $0.5 \mathrm{~s}$ period before onset of thrusting. Period 3 was defined as the period between Periods 2 and 4 . Period 5 was defined as the thrust period. Period 6 was defined as the $0.25 \mathrm{~s}$ period after offset of thrusting. $\boldsymbol{B}$, Definition of the periods for genital grooming. The same baseline period as in $\boldsymbol{A}$ was used because genital grooming was induced after thrusting, intromission, or ejaculation. Periods 1 and 2 were defined as the $0.5 \mathrm{~s}$ periods before and after onset of genital grooming, respectively. Periods 4 and 5 were defined as the $0.5 \mathrm{~s}$ periods before and after offset of genital grooming, respectively. Period 3 was defined as the period between Periods 2 and 4. C, Definition of the periods for sniffing inaccessible females. Periods $1-5$ were similarly defined as those in $\boldsymbol{B}$. A baseline period was defined as the period from -3.5 s to $-0.5 \mathrm{~s}$ before onset of sniffing. amygdala (Brog et al., 1993), which are involved in copulation performance (Hull and Rodríguez-Manzo, 2009). Two major neuronal types have been identified in the NAc (Meredith, 1999): fast spiking interneurons (FSIs) and medium spiny projection neurons (MSNs). FSIs strongly inhibit MSNs and control their spike timing (Tepper and Plenz, 2006) and responded differently than MSNs to rewards (Lansink et al., 2010), suggesting that FSIs and MSNs play different roles in sexual behavior. In this study, we investigated neuronal activities (FSIs and MSNs) in the NAcS of male rats during sexual behavior.

\section{Materials and Methods \\ Subjects}

Fifteen adult male Wistar rats weighing 300 $430 \mathrm{~g}$ (SLC) were used for this study. Housing temperature was maintained at $23 \pm 1^{\circ} \mathrm{C}$ with a $12 \mathrm{~h}$ light/dark cycle. Before surgery, two male rats were housed per cage, whereas after surgery, they were housed individually, with food and water available ad libitum. Sixteen adult female rats weighing $230-330 \mathrm{~g}$ (SLC), housed two rats per cage with food and water available ad libitum, were used as stimuli for male copulatory behavior. All rats were treated in strict compliance with the United States Public Health Service Policy on Human Care and Use of Laboratory Animals, National Institutes of Health Guide for the Care and Use of Laboratory Animals, and Guidelines for the Care and Use of Laboratory Animals at the University of Toyama, and all experimental procedures were approved by our institutional committee for experimental animal ethics. Every attempt was made to minimize the number of animals used and their suffering.

\section{Surgery}

Stimulus adult female rats were ovariectomized under intraperitoneal sodium pentobarbital anesthesia $(40 \mathrm{mg} / \mathrm{kg})$. The male rats were trained before surgery, and those that fulfilled a certain criterion were operated 
upon (for details, refer to Training). Rats were anesthetized intraperitoneally with sodium pentobarbital $(40 \mathrm{mg} / \mathrm{kg})$, and a recording electrode assembly was implanted in the upper part of the left NAcS (1.5-1.9 mm rostral from the bregma, $1.3 \mathrm{~mm}$ lateral from the midline, and $7.3 \mathrm{~mm}$ below the brain surface) after referring to the atlas of Paxinos and Watson (2007). The recording electrode assembly comprised 4 tetrodes, each of which included four tungsten microwires (20 $\mu \mathrm{m}$ in diameter; California Fine Wire), which were encased in a stainless steel cannula (30 gauge; Hakko), and a microdrive. The tip impedance was $\sim 200 \mathrm{k} \Omega$ at $1 \mathrm{kHz}$.

\section{Experimental setup}

A testing chamber made of transparent acrylic was used for recording. It comprised two compartments (large, $30 \times 39 \times 39 \mathrm{~cm}$; small, $17 \times$ $39 \times 39 \mathrm{~cm}$ ) separated by removable double stainless mesh walls with a $2 \mathrm{~cm}$ interval. The double stainless mesh walls prevented physical contact between rats. The analog signals of neuronal activities were digitized and stored in a computer via a Multichannel Acquisition Processor system (MAP: Plexon). The amplified neuronal signals were digitized at a $40 \mathrm{kHz}$ sampling rate, and $1.2 \mathrm{~ms}$ waveforms that crossed an experimenter-defined threshold were stored on a computer hard disk for offline spike sorting. The threshold was set to a level at which only a few noises crossed (see the manual by Plexon; http://www. plexon. com/ assets/downloads/RASPUTINV2Manual.pdf). Rat behaviors were captured at 20 frames/s by a charged-coupled device (CCD) camera and stored on a hard disk using the CinePlex program (Plexon), which synchronized the video images with the neuronal data. The video was captured obliquely from above the chamber. To capture both the left and right sides of the rat bodies, a mirror was placed behind the chamber, and the images in the mirror were simultaneously captured using the same CCD camera.

\section{Training}

Both training and recording were conducted between 9:00 P.M. and 12:00 A.M. in the dark phase. Female rats were subcutaneously injected with estradiol benzoate $(5 \mu \mathrm{g} / \mathrm{rat})$ and progesterone $(500 \mu \mathrm{g} / \mathrm{rat}) 48 \mathrm{~h}$ and $4-7 \mathrm{~h}$, respectively, before training or recording. Before surgery, the male rats were trained 3 times for copulatory behavior at $>4 \mathrm{~d}$ intervals. In each training session, the male rat could freely interact with the estrous female for 60-120 min in a transparent acrylic chamber $(49 \times 39 \times 39 \mathrm{~cm})$. Only those rats that ejaculated at least three times within the first $60 \mathrm{~min}$ in the last training session were selected for surgery and recording.

\section{Behavioral and recording procedures}

The male rat was placed in the large compartment of the test chamber, and neuronal activity was checked daily. If stable neuronal signals over 10 min were found and if $>4 \mathrm{~d}$ had passed since the last previous recording day, the recording experiment described below was conducted. If signals were found before the interval of $4 \mathrm{~d}$, the male rat was returned to his home cage. If no signal was found, the electrode assembly was lowered by $\sim 22-88 \mu \mathrm{m}$ and the male rat was returned to the home cage.

The recording experiment included the following session comprising four phases, and was conducted three times (Fig. 1). In Phase 1, the male rat was placed alone in the large compartment for $5 \mathrm{~min}$. In Phase 2, the estrous female rat was placed for $5 \mathrm{~min}$ in the small compartment, which was separated from the large compartment by a wall (presentation of the female rat). This allowed the male and the female to interact without physical contact. In Phase 3, the wall was removed and the rats were allowed to freely interact with each other and copulate. Phase 4 (postejaculation period) was defined as the period ( $1 \mathrm{~min}$ ) after ejaculation. At the end of Phase 4, the female was removed from the chamber, the male rat was returned to the larger compartment, and the walls were reinserted. After an interval of $9 \mathrm{~min}$, the same session was repeated three times. After the three recording sessions were completed, the recording sessions were terminated on that day. Then, the electrode assembly was lowered by at least $44 \mu \mathrm{m}$ to record new neuron(s) in the next recording sessions, and the male rat was returned to his home cage.

\section{Data analysis}

Spike sorting and classification of neurons. The digitized neuronal activity was isolated into single units by waveform components using the Offline 

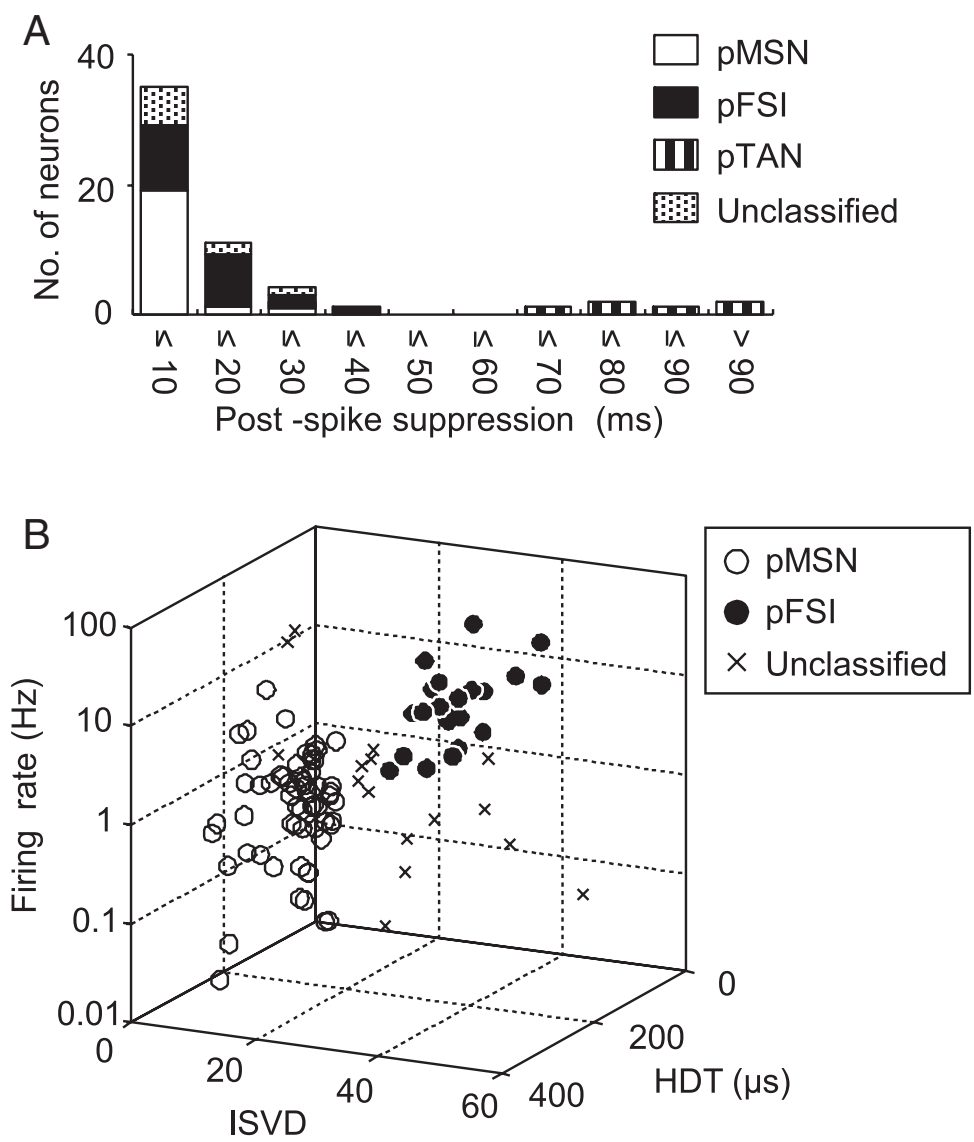

C pMSN

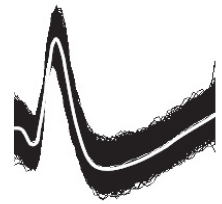

pFSI

$500 \mu \mathrm{s}$
Previous studies have reported that distinct types of striatal neurons responded differently to water and food rewards (Berke, 2008; Lansink et al., 2010). To examine whether such response patterns also exist for sexual rewards, each neuron was classified on the basis of its electrophysiological properties. According to previous neurophysiological studies in the rat striatum (Berke et al., 2004; Berke, 2008; Schmitzer-Torbert and Redish, 2008; Lansink et al., 2010), the following four parameters were checked to classify NAcS neurons: (1) postspike suppression (Schmitzer-Torbert and Redish, 2008), the period that passed before neuronal activity returned to its average firing rate after each action potential; (2) initial slope of valley decay (ISVD) of the waveform (Lansink et al., 2010); (3) valley half decay time (HDT) of the waveform (Lansink et al., 2010); and (4) mean firing rate. Putative tonically active neurons (pTANs), which were expected to be cholinergic interneurons, were defined as such if their postspike suppression was $>50 \mathrm{~ms}$ and mean firing rates were $>2 \mathrm{~Hz}$. Putative fast spiking interneurons ( $\mathrm{pFSI}$ ) were defined as such if their mean firing rates were $>2 \mathrm{~Hz}$, ISVD was $>22$, HDT was $<250 \mu$ s, and postspike suppression was $<50 \mathrm{~ms}$. Putative medial spiny projection neurons ( $\mathrm{pMSNs}$ ) were defined as such if their ISVD was $<22$, HDT was $>240 \mu \mathrm{s}$, and postspike suppression was $<50$ $\mathrm{ms}$. The neurons that did not match any of the above criteria were defined as unclassified neurons (UN).

Correlation between neuronal activity and behavior. CinePlex program (Plexon) was used to play back the recorded video, and timings of four behaviors were determined, which included onset and offset of copulatory behavior (Phase 3), onset and offset of genital auto-grooming (Phases 3 and 4, and very rarely Phase 2), onset and offset of sniffing female odor across the wall (Phase 2), and onset of chasing behavior before copulatory behavior (Phase 3). Copulatory behavior was further classified into 3 types: mount + thrust (thrusting without penile insertion after mounting), intromission + thrust (thrusting with penile insertion), and ejaculation + intromission + thrust (thrusting with intromission followed by ejaculation). These three types of copulatory behavior always occurred after chasing behavior. Next,

Figure 4. Neurophysiological classification of NACS neurons. A, Postspike suppression histogram of NAcS neurons. Ordinate and abscissa indicate the number of NACS neurons and the postspike suppression period in $10 \mathrm{~ms}$ steps, respectively. Neurons with firing rates $<2 \mathrm{~Hz}$ were not analyzed. The cluster of pTANs with a long postspike suppression period is identified. $\boldsymbol{B}$, Scatter plot of NACS neurons based on mean firing rates and waveform properties (ISVD and HDT). The clusters of pMSNs and pFSIs are identified. C, Examples of superimposed waveforms of a pMSN (left) and a pFSI (right).

Sorter program (Plexon). Superimposed waveforms of the isolated units were drawn to check their consistency throughout the recording sessions, and were then transferred to the NeuroExplorer program (Nex Technology) for further analysis. Typically, 1-4 single units were isolated by offline cluster analysis from four channels (wires) of one tetrode. Spike sorting was performed with the Offline Sorter program (Plexon) that can plot spikes in two- or three-dimensional feature spaces, in which various features of spike waveforms (waveform projection onto principal components, peak amplitudes of the waveforms, valley amplitudes of the waveforms, peak-valley amplitudes of the waveforms, etc.) can be selected as a dimension. Each cluster in the feature space was then checked manually to ensure that the cluster boundaries were well separated and the waveform shapes were consistent with the action potentials. For each isolated cluster, an interspike interval histogram was constructed and an absolute refractory period of at least $1.0 \mathrm{~ms}$ was used to exclude suspected multiple units. Finally, superimposed waveforms of the isolated units were drawn to check waveform consistency. Thus, 1-11 single units were recorded from four tetrodes for each rat per experimental session (day). perievent histograms aligned with onset and/or offset of the above behaviors were created; however, neuronal correlates with mount + thrust and intromission + thrust + ejaculation were not analyzed because the number of these events in one recording experiment was usually low.

Figure 2 illustrates the definition of behavioral periods for the statistical analyses of neuronal responses to chasing and copulatory behaviors $(A)$, genital grooming $(B)$, and sniffing of inaccessible females $(C)$. Because copulatory behavior (mount + thrust, intromission + thrust, and ejaculation + intromission + thrust) always occurred after chasing behavior, each copulatory behavior was analyzed with chasing $(A)$. Seven behavioral periods were defined to analyze neuronal responses to these behaviors: baseline period for $3 \mathrm{~s}, 0.5 \mathrm{~s}$ period before onset of chasing behavior (Period 1), chasing period (Periods 2-4), copulatory period (Period 5), and $0.25 \mathrm{~s}$ period after offset of copulatory behavior (Period 6). The chasing period was further divided into three periods: $0.5 \mathrm{~s}$ period after onset of chasing behavior (Period 2), $0.5 \mathrm{~s}$ period before offset of chasing behavior (Period 4), and mid- 


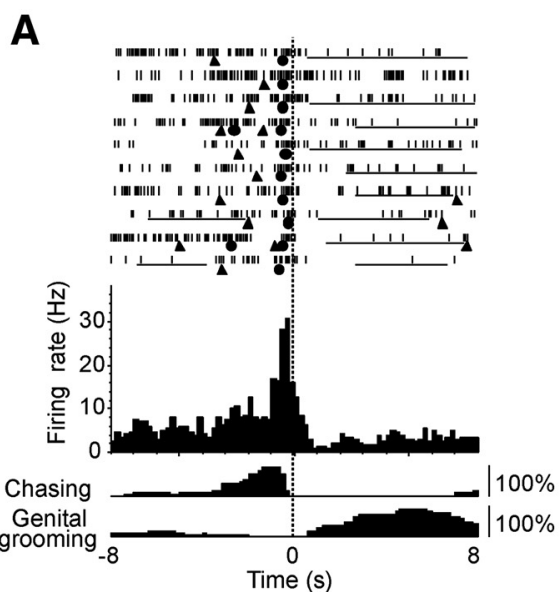

C

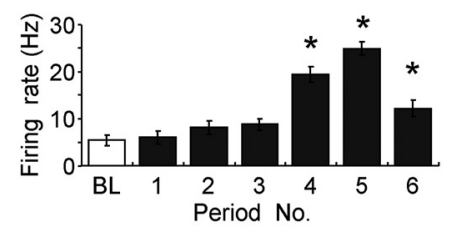

B

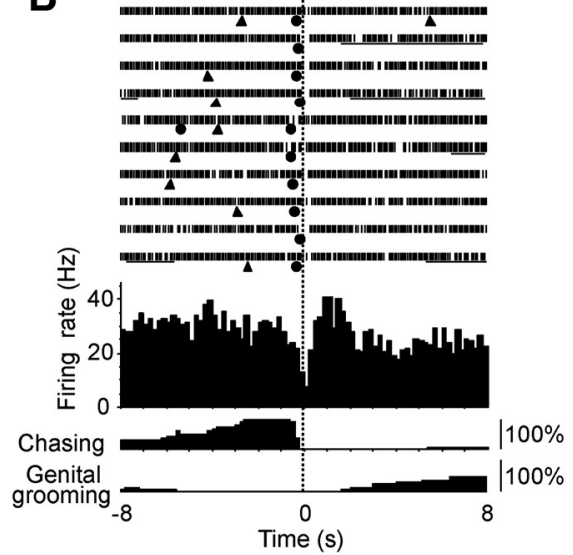

D

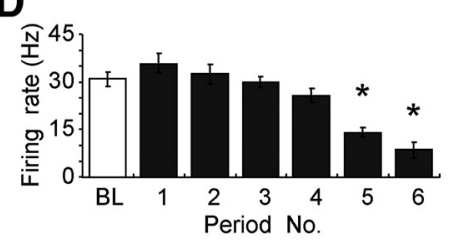

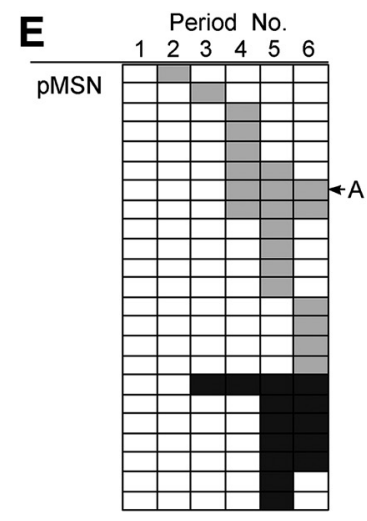

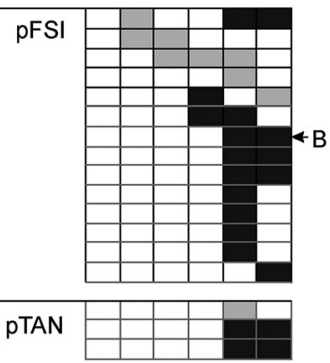

$\mathbf{F}$
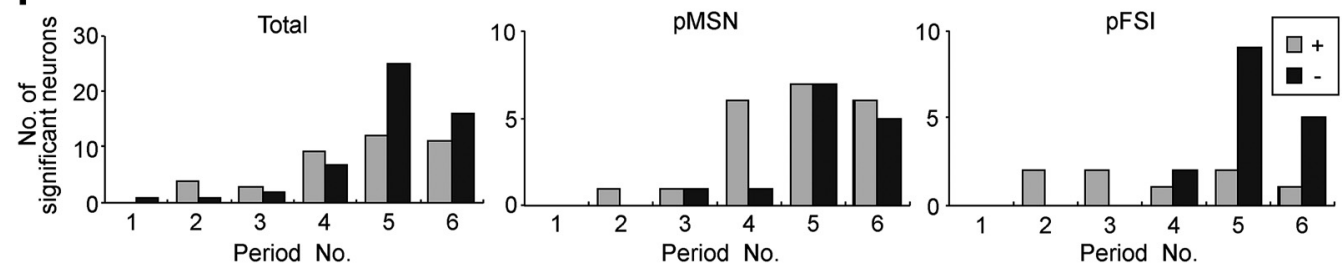

UN

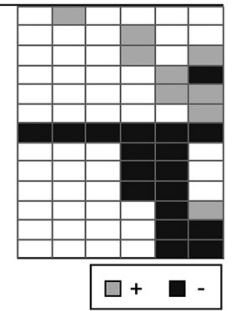

Figure 5. Neuronal responses to pelvic thrusting with intromission. $\boldsymbol{A}, \boldsymbol{B}$, Perievent rasters and histograms (bin width $=0.2 \mathrm{~s}$ ) of pMSN responses $(\boldsymbol{A})$ and pFSI responses $(\boldsymbol{B})$. Top, middle, and bottom histograms represent neuronal firings and frequency of chasing and genital grooming per bin, respectively. Time 0 indicates offset of thrusting with intromission. Triangles: onset of chasing, circles: onset of thrusting, underlines: duration of genital grooming. $\boldsymbol{C}, \boldsymbol{D}$, Mean firing rates of the pMSN shown in $\boldsymbol{A}(\boldsymbol{C})$ and the pFSI shown in $\boldsymbol{B}(\boldsymbol{D})$ at baseline and in Periods $1-6$. *Significant difference from the baseline (Bonferroni correction, $p<0.05$ ). $\boldsymbol{E}$, Response patterns of all neurons that responded to intromission and thrusting. Each horizontal line comprising six squares represents responses of each neuron in Periods 1-6 (P1-6). Gray and black squares indicate excitatory and inhibitory responses. $\boldsymbol{F}$, Number of neurons with excitatory (gray) or inhibitory (black) responses in each period in all recorded neurons (left), pMSNs (middle), and pFSIs (right).

interval between these two periods (Period 3). Genital grooming was divided into five periods (B): $0.5 \mathrm{~s}$ period before onset of genital grooming (Period 1), $0.5 \mathrm{~s}$ period after onset of genital grooming (Period 2), 0.5 s period before offset of genital grooming (Period 4$), 0.5$ s period after offset of genital grooming (Period 5), and mid-interval between Periods 2 and 4 (Period 3). The same baseline period as that shown in Figure $2 \mathrm{~A}$ was used in this analysis because genital grooming mostly occurred after copulatory behavior. Sniffing was divided into 6 periods $(C)$ : baseline period for $3 \mathrm{~s}, 0.5 \mathrm{~s}$ period before onset of sniffing (Period 1 ), $0.5 \mathrm{~s}$ period after onset of sniffing (Period 2), $0.5 \mathrm{~s}$ period before offset of sniffing (Period 4), $0.5 \mathrm{~s}$ period after offset of sniffing (Period 5), and midinterval between Periods 2 and 4 (Period 3). The average firing rates in these behavior-defined periods were compared with the baseline activity by Bonferroni tests (post hoc tests) after repeated measures one-way ANOVA. Differences were considered to be significant if the $p$-value was $<0.05$ and the average difference was $>1.0 \mathrm{~Hz}$. Responses of each neuron to a given behavior were classified into four categories on the basis of post hoc comparisons with the baseline activity: excitatory (if the firing rate increased in at least 1 period), inhibitory (if the firing rate decreased in at least 1 period), both excitatory and inhibitory, and no response. Statistical significance of the ratios of excitatory and inhibitory neurons were assessed by the $\chi^{2}$ test $(\mathrm{df}=1)$ with a significance level of $p<0.05$. Ratios of excitatory and inhibitory responses were also compared between pMSNs and pFSIs. pTANs were excluded from this analysis because the total number of recorded pTANs was low.
Because the three types of copulatory behavior included the same action (thrusting), activities in the prethrust and thrust periods across the three types were compared. Since actions after thrusting were completely different among these three types of copulatory behavior, neuronal activities in the post-thrust period were not compared. First, in each NAcS neuron, activity in the thrust period of intromission + thrust (Fig. $2 \mathrm{~A}$, Period 5) was compared with that in the thrust period of the other two types by the unpaired $t$ test. Differences were considered to be significant if the $p$ value was $<0.05$ and the average difference was $>1.0 \mathrm{~Hz}$. Neuronal activities during mount + thrust and ejaculation + intromission + thrust were not compared because both the behaviors were usually low in frequency. Second, neuronal activity in the prethrust period (Fig. $2 \mathrm{~A}$, Period 4) of intromission + thrust was compared with that in the prethrust period of each of the other two copulatory behavior by the unpaired $t$ test $(p<0.05)$.

Comparison of average firing rates across the different phases. To analyze responses to phase-specific cues or contexts, average firing rates in the four phases were compared in each neuron. The average firing rate in each phase was calculated in each session. The data in the periods during which specific behaviors were observed (Fig. 2 A, Periods 1-6; $B$, Periods $1-5$; $C$, Periods $1-5$ ) were excluded from this analysis. The average firing rates were then compared among the four phases by repeated-measures ANOVA and a post hoc test (Tukey's test) with a significance level of $p<0.05$. 
A
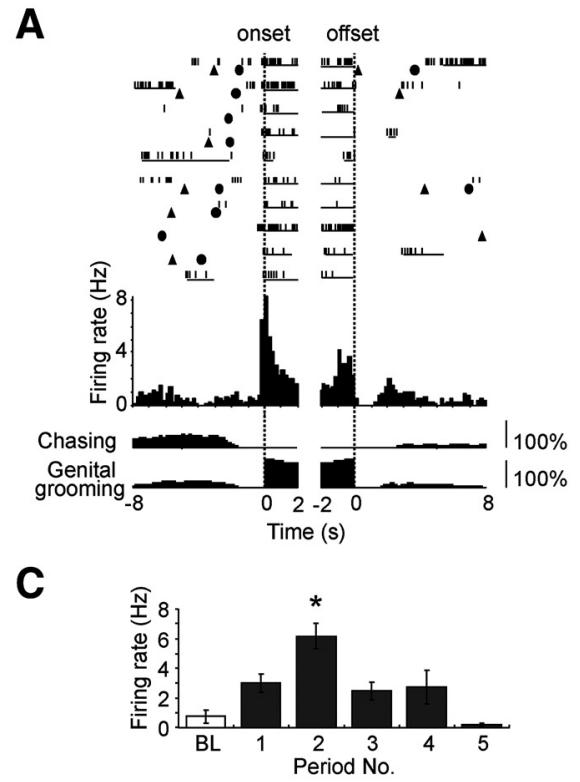

B

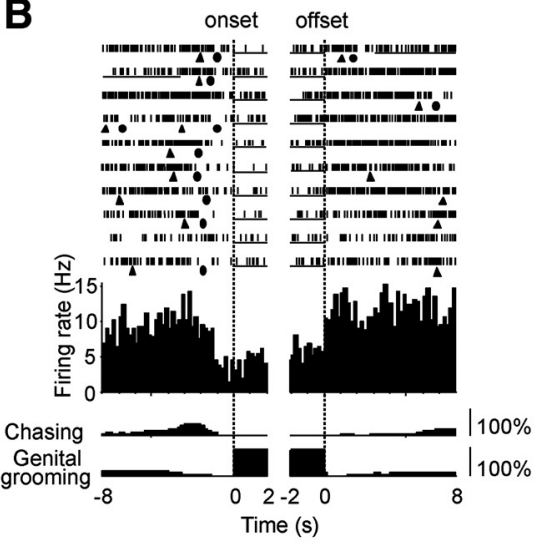

D

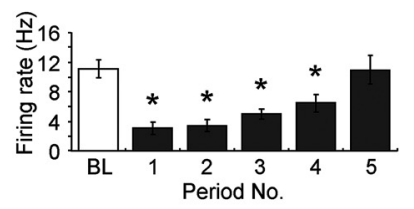

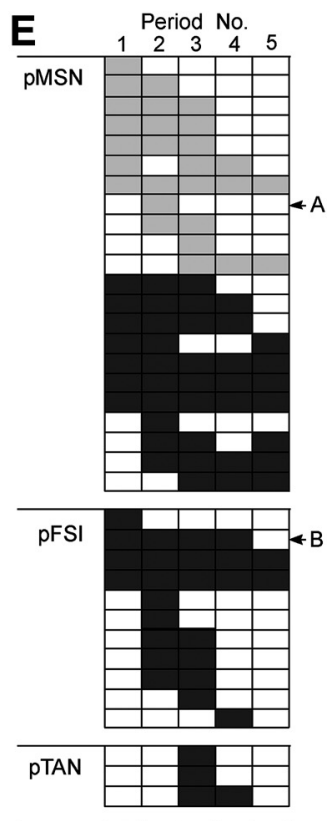

UN
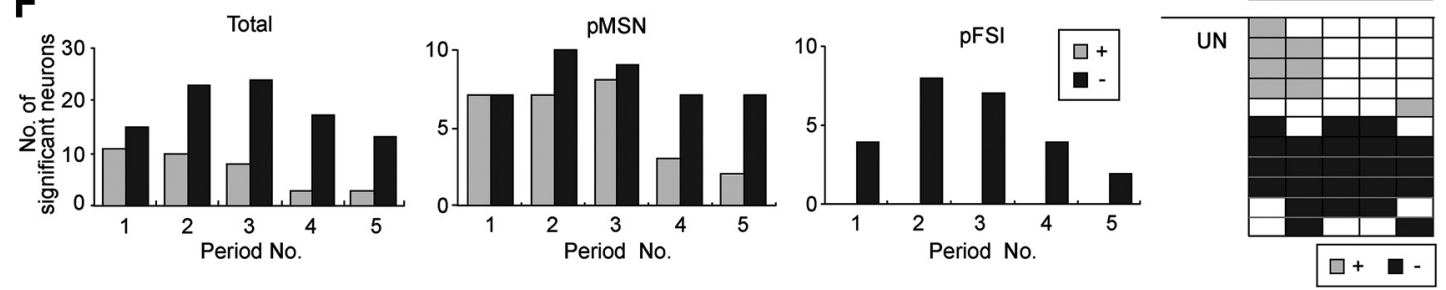

Figure 6. Neuronal responses to genital grooming. $\boldsymbol{A}, \boldsymbol{B}$, Perievent rasters and histograms (bin width $=0.2 \mathrm{~s}$ ) of responses of a pMSN $(\boldsymbol{A})$ and a pFSI (B). Top, middle, and bottom histograms represent neuronal firings and frequency of chasing and genital grooming per bin, respectively. Time 0 in the left and right histograms indicates onset and offset of genital grooming, respectively. Triangles: onset of chasing, circles: onset of thrusting, underlines: duration of genital grooming. $\boldsymbol{C}, \boldsymbol{D}$, Mean firing rates of the pMSN shown in $\boldsymbol{A}(\boldsymbol{C})$ and the pFSI shown in $\boldsymbol{B}(\boldsymbol{D})$ at baseline and in Periods 1-5. *Significant difference from baseline (Bonferroni correction, $p<0.05$ ). $\boldsymbol{E}$, Response patterns of all neurons that responded to genital grooming. Each horizontal line comprising five squares represents responses of each neuron in Periods 1-5 (P1-5). Gray and black squares indicate excitatory and inhibitory responses. $F$, Number of neurons with excitatory (gray) or inhibitory (black) responses in each period in all recorded neurons (left), pMSNs (middle), and pFSIs (right).

Periodicity of firings. Periodic firing patterns in the $1-20 \mathrm{~Hz}$ range during copulatory behaviors were analyzed. An auto-correlogram over $2 \mathrm{~s}$ (bin size $=1 \mathrm{~ms}$ ) was calculated in each phase and filtered with the Gaussian filter (full width at half maximum, $10 \mathrm{~ms}$ ). Then, according to König (1994) and Engel et al. (1990), the primary oscillation frequency between 1 and $20 \mathrm{~Hz}$ was calculated by nonlinear fitting of the following function to the auto-correlogram.

$$
f(t)=A \cdot \exp \left(\left(-t / \sigma_{1}\right)^{2}\right) \cdot \cos (2 \pi v(t))+O+B \cdot \exp \left(\left(-t / \sigma_{2}\right)^{2}\right),
$$

where the first term represents Gabor function; the second term $(O)$ is an offset; the third term represents a Gaussian function to consider a central modulation of the auto-correlogram; $t$ is time; $A, \sigma_{1}$, and $\nu$ are amplitude, decay constant, and wave frequency of the Gabor function, respectively; and $B$ and $\sigma_{2}$ are amplitude and width of the Gaussian function, respectively. Frequency of oscillation of a given neuron corresponds to wave frequency of the Gabor function $(\nu)$. Although the same function and the algorithm for nonlinear regression were used as reported by König (1994), the criteria were slightly modified. A given neuron was considered to be significantly oscillated in a given frequency $(\nu)$ in a given phase according to the following three criteria: $(1)$ the function was regressed with the effective coefficient of the amplitude $(A)$ and frequency $(\nu)(p<0.05),(2)$ the decay constant $\left(\sigma_{1}\right)$ was larger than $1 / \nu^{\star} 0.8$, which means that the fitted function had at least one satellite peak (Engel et al., 1990), and (3) the number of spikes within the auto-correlogram was $>150$.

The following four frequency ranges of oscillation were defined: delta $(1-4 \mathrm{~Hz})$, low theta $(4-7 \mathrm{~Hz})$, high theta $(7-12 \mathrm{~Hz})$, and alpha $(12-20 \mathrm{~Hz})$. The number of neurons with significant oscillation in each frequency range in each phase was counted. Then, in each frequency range, the ratio of the number of neurons with significant oscillation in the given frequency range to the number of neurons with $>150$ spikes within the auto-correlogram of each phase were statistically assessed by the $\chi^{2}$ test $(\mathrm{df}=3$ ) with a significance level of $p<0.05$ and residual analysis with a significance level of standard residual $>2.0$.

Similarly, low gamma $(40-60 \mathrm{~Hz})$ and high gamma $(60-80 \mathrm{~Hz})$ range oscillation were analyzed by computing narrower auto-correlograms over $0.2 \mathrm{~s}$ (bin size $=0.1 \mathrm{~ms}$ ), which were filtered by the Gaussian filter (full width at half maximum, $1 \mathrm{~ms}$ ).

Behavioral activity around ejaculation. After ejaculation, male rats become sexually and behaviorally quiescent for several minutes (̊ggmo, 2007; Hull and Rodríguez-Manzo, 2009). This period is called the postejaculatory interval. To confirm that the rats also entered this period in the present study, behavioral activity of the rats before and after ejaculation was analyzed. For this purpose, activity index was defined as the mean frequency of switching body direction (left to right or right to left) per min, and the activity index was compared between Phases 3 and 4 (i.e., before and after ejaculation).

Histology. After the recording, rats were deeply anesthetized with intraperitoneal pentobarbital sodium $(50 \mathrm{mg} / \mathrm{kg})$, and the recording sites were marked by electrolytic lesions by passing a $20-\mu \mathrm{A}$ negative current through the recording electrodes for $30 \mathrm{~s}$. The rats were then perfused through the heart with $0.9 \%$ saline followed by $10 \%$ buffered formalin containing $2 \%$ potassium ferricyanide. The brain was removed and fixed in formalin for at least $48 \mathrm{~h}$. Serial sections of $60 \mu \mathrm{m}$ were cut on a freezing microtome and stained with Cresyl Violet. 
A
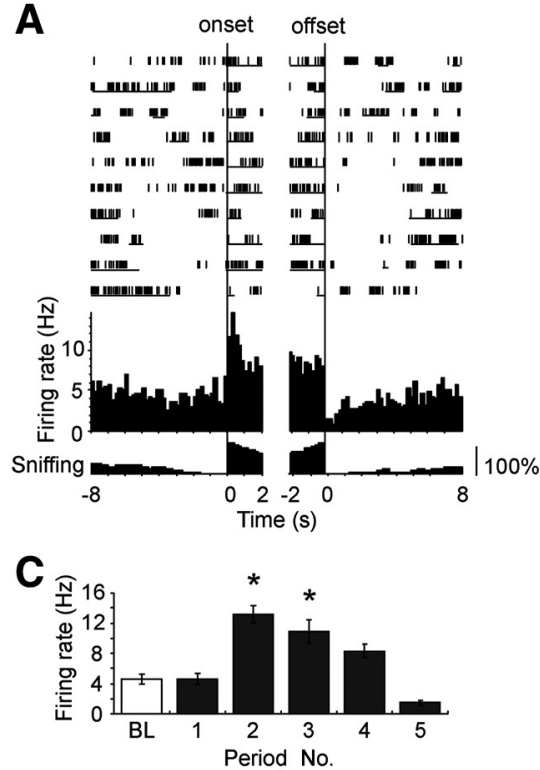

B

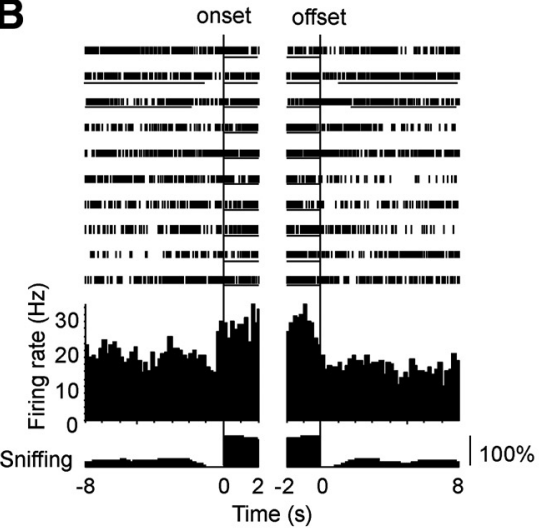

D

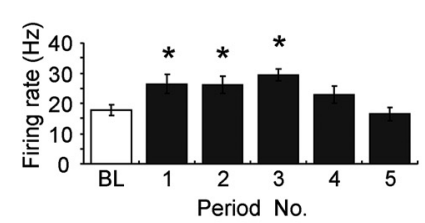

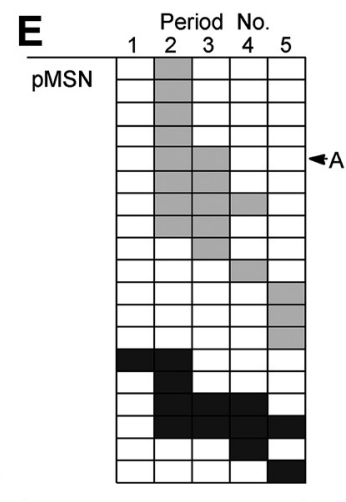

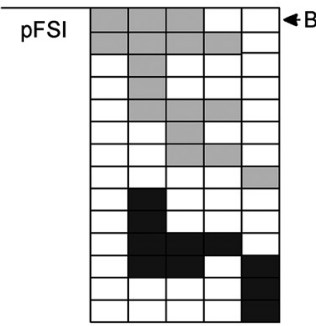

$\mathbf{F}$
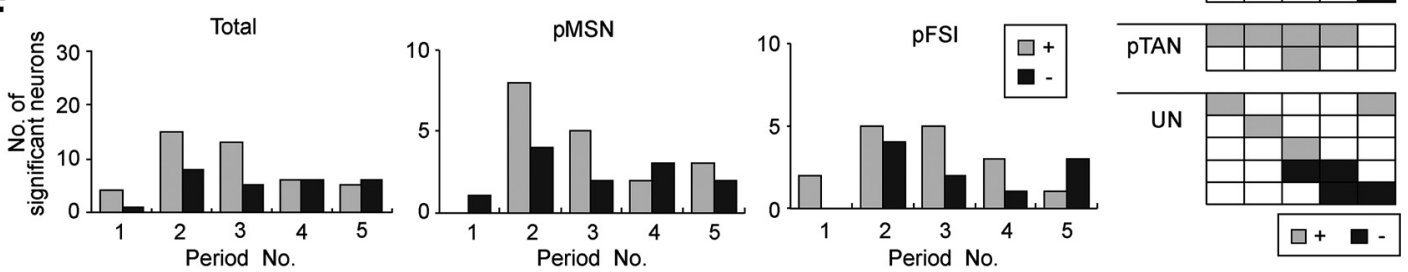

Figure 7. Neuronal responses to sniffing of females in Phase 2. $\boldsymbol{A}, \boldsymbol{B}$, Perievent rasters and summed histograms (bin width $=0.2 \mathrm{~s}$ ) of responses of a pMSN $(\boldsymbol{A})$ and a pFSI $(\boldsymbol{B})$. Top and bottom histograms represent neuronal firings and frequency of sniffing per bin, respectively. Time 0 in the left and right histograms indicates onset and offset of sniffing, respectively. Underlines under the raster indicate duration of sniffing. $\boldsymbol{C}, \boldsymbol{D}$, Mean firing rates of the pMSN shown in $\boldsymbol{A}(\boldsymbol{C})$ and the pFSI shown in $\boldsymbol{B}(\boldsymbol{D})$ at baseline and in Periods $1-5$. * Significant difference from the baseline (Bonferroni correction, $p<0.05)$. $\boldsymbol{E}$, Response patterns of all neurons that responded to sniffing. Each horizontal line comprising five squares represents responses of each neuron in Periods $1-5$ (P1-5). Gray and black squares indicate excitatory and inhibitory responses. $\boldsymbol{F}$, Number of neurons with excitatory (gray) or inhibitory (black) responses in each period in all recorded neurons (left), pMSNs (middle), and pFSIs (right).

\section{Results}

Neurophysiological classification of NAcS neurons

Stable activities of 123 NAcS neurons across the three sessions were recorded from 15 rats. Typical waveforms of four NAcS neurons $(a-d)$, which were simultaneously recorded from the same tetrode (EL 1-4), are shown in Figure 3A. Figure $3 B$ shows the results of spike sorting by offline cluster cutting of the neural activities shown in Figure 3A. Each dot represents one spike, and four clusters of dots indicated by different colors were recognized. Units $a-d$ in Figure $3 B$ represent the four single units ( $a-d$, respectively) shown in Figure 3A. Figure 4 shows three types of NAcS neurons based on neurophysiological parameters. pTANs exhibited longer postspike suppression periods than did pMSNs and pFSIs (Fig. 4A), which enabled discrimination of pTANs from pMSNs and pFSIs. A scatter plot of the remaining NAcS neurons, other than the pTANs, based on the neurophysiological criteria of mean firing rates and waveform properties (ISVD and HDT) indicated that clusters of pMSNs (open circles) and pFSIs (closed circles) were clearly identified (Fig. 4B). Thus, 60, 21, and 6 neurons were classified as pMSNs, pFSIs, and pTANs, respectively, and 36 neurons were unclassified. Examples of superimposed waveforms of a pMSN and a pFSI are shown in Figure 4C.

Neuronal correlates with sexual behaviors

Firing changes associated with intromission

Fifty-three $(43 \%, 53 / 123)$ neurons exhibited significantly changed firing rates in at least one of the defined periods of intromission + thrust (behavior-responsive neurons). Of these, 25 exhibited excit- 


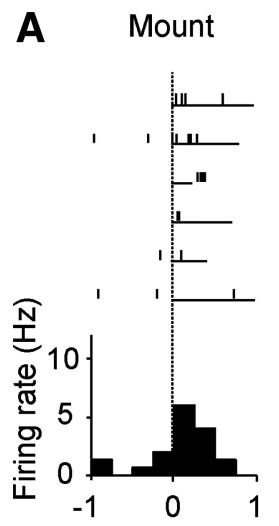

C
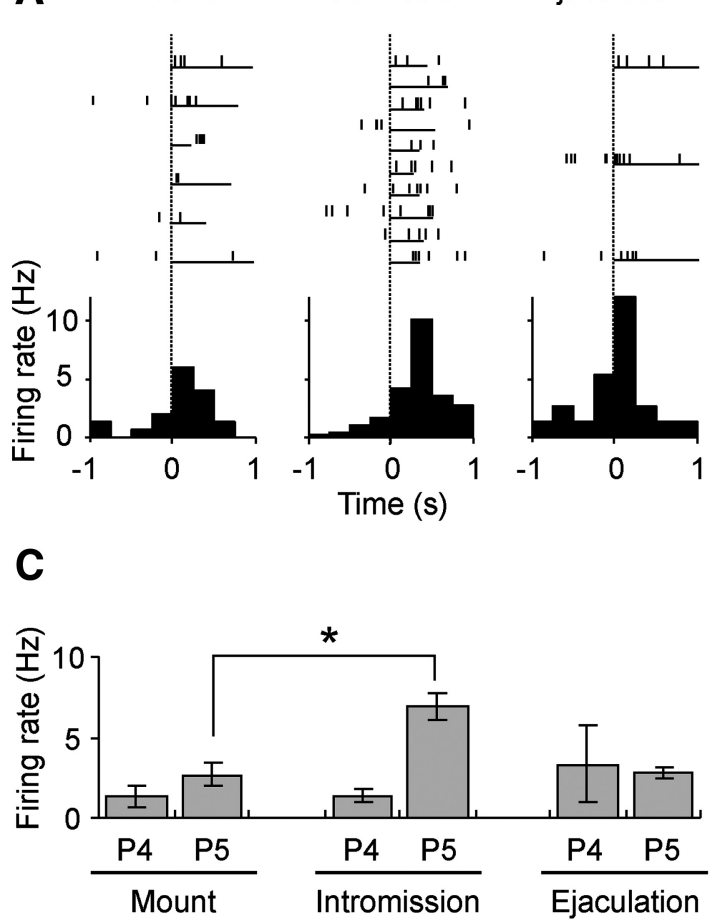

B

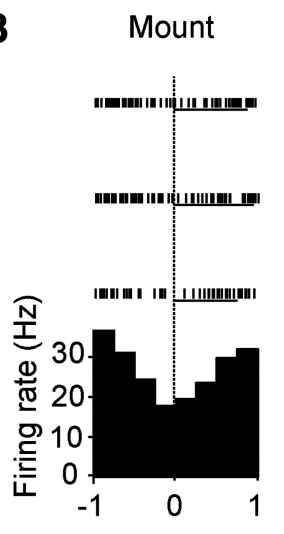

Ejaculation

D

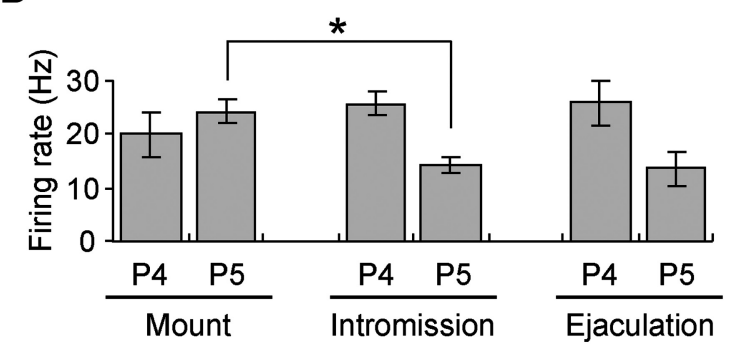

Figure 9. Examples of NACS neurons with differing responses to the three copulatory behaviors. $\boldsymbol{A}, \boldsymbol{B}$, Perievent raster and summed histograms (bin size $=0.25 \mathrm{~s})$ of responses of a pMSN $(\boldsymbol{A})$ and a pFSI $(\boldsymbol{B})$ to the three types of copulatory behavior (mount + thrust, intromission + thrust, ejaculation + intromission + thrust). Rasters and summed histograms are aligned with onset of thrusts. $\boldsymbol{C}, \boldsymbol{D}$, Mean firing rates of the pMSN $(\boldsymbol{C})$ and pFSI $(\boldsymbol{D})$ in Periods 4 and 5 in each copulatory behavior. Responses in $\boldsymbol{A}, \boldsymbol{C}$ and $\boldsymbol{B}, \boldsymbol{D}$ were recorded from the same neurons. ${ }^{*}$ Significant difference (unpaired $t$ test, $p<0.05$ ).

atory responses, 24 exhibited inhibitory responses, and 4 exhibited both excitatory and inhibitory responses. Examples of pMSN and pFSI responses to intromission + thrust are shown in Figure 5, A and $B$, respectively. The rasters and summed histograms of neuronal activity were aligned with offset of thrusting (Fig. 5A,B). Triangles, circles, and underlines under the rasters indicate onset of chasing, onset of thrusting, and duration of genital grooming, respectively. Activity of the pMSN gradually increased during thrusting (Fig. 5A), while activity of the pFSI gradually decreased during thrusting (Fig. $5 B)$. The mean firing rates of these two neurons at baseline and in Periods 1-6 are shown in Figure 5, $C$ and D, respectively. Activity of the pMSN significantly increased in Periods 4-6 (excitatory response) (Fig. 5C) (Bonferroni test after repeated measures one-way ANOVA, $p<0.05$ ), while activity of the pFSI significantly decreased in Periods 5-6 (inhibitory response) (Fig. 5D) (Bonferroni test after repeated measures one-way ANOVA, $p<0.05$ ). Figure $5 E$ shows response patterns of all the responsive NAcS neurons across the six behavioral periods, and the number of NAcS neurons with significant firing changes in each period is shown in Figure $5 F$. These results indicate that the NAcS neurons responded to intromission + thrust at various times, and that most pFSIs were inhibited after onset of thrusting.

\section{Firing changes associated with genital grooming}

Genital grooming was observed mostly after intromission in the present study. Penis temperature seemed to be higher after intromission than outside the context of sexual interaction due to rubbing in the vagina and higher intracavernous pressure during intromission versus noncontact erection outside the context of sexual interaction (Bernabé et al., 1999). Furthermore, when the penis was warmer, afferent sensory fibers in the dorsal penile nerve were more sensitive to mechanical stimulation (Johnson and Kitchell, 1987). These findings suggest that the dorsal penile nerve is more sensitive to genital grooming after intromission than outside the context of sexual interaction.

In this study, 47 (38\%, 47/123) neurons exhibited significantly changed firing rates in at least one of the defined periods of genital grooming (behavior-responsive neurons). Of these, 16 and 31 neurons exhibited excitatory and inhibitory responses, respectively. Examples of pMSN and pFSI responses to genital grooming are shown in Figure 6, $A$ and $B$, respectively. The rasters and summed histograms of neuronal activity were aligned with onset and offset of genital grooming (Fig. 6A,B). Triangles, circles, and underlines under the rasters indicate onset of chasing behavior, onset of thrusting, and duration of genital grooming, respectively. Activity of the pMSN was increased after onset of genital grooming (Fig. 6A), while activity of the pFSI was decreased before and after onset of genital grooming (Fig. $6 B$ ). The mean firing rates of these neurons at baseline and in Periods $1-6$ are shown in Figure 6, $C$ and $D$. Activity of the pMSN significantly increased in Period 2 (Bonferroni test after repeated measures one-way ANOVA, $p<0.05$ ) (Fig. 6C), while activity of the pFSI significantly decreased in Periods 1-4 (Bonferroni test after repeated measures one-way ANOVA, $p<0.05$ ) (Fig. 6D). Figure $6 E$ shows response patterns of all the responsive NAcS neurons across the five behavioral periods, and the number of NAcS neurons with significant firing changes in each period is shown in Figure $6 \mathrm{~F}$. These results indicate that the NAcS neurons responded to genital grooming at various times, and that all responsive $\mathrm{pF}$ SIs were inhibited around the time of genital grooming.

Firing changes associated with sniffing of females

Forty $(33 \%, 40 / 123)$ neurons exhibited significantly changed firing rates in at least one of the defined periods of sniffing (behavior- 
Table 1. Number of neurons with different firing rates between two different copulatory behaviors

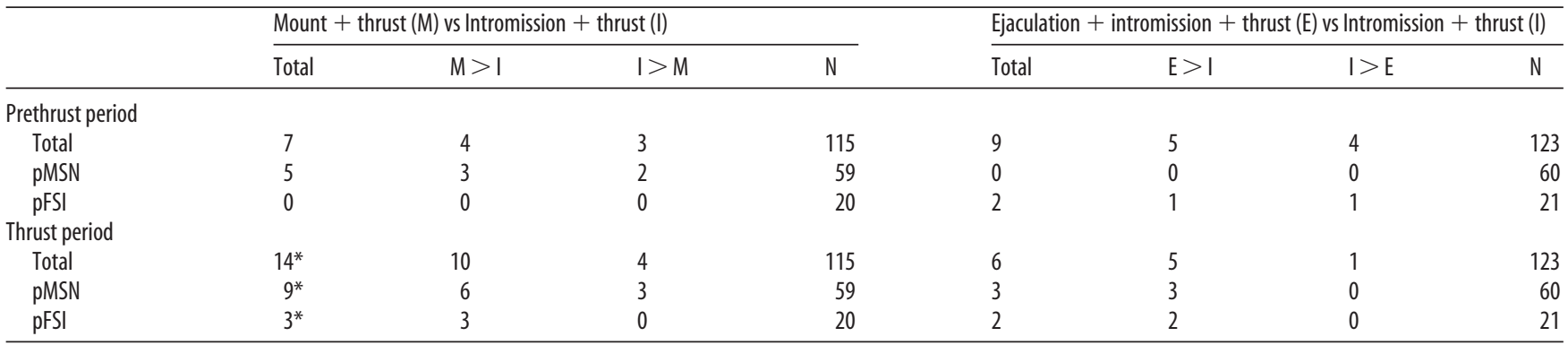

$M$, Thrust after mount (mount + thrust); l, thrust with intromission (intromission + thrust); E, thrust with intromission followed by ejaculation (ejaculation + intromission + thrust); $N$, number of recorded neurons. Asterisks indicate that the number is significantly larger than chance level ( $5 \%$, which is the type I error rate of the $t$ test used for the comparisons) $\left(\chi^{2}\right.$ test, $\left.p<0.05\right)$.
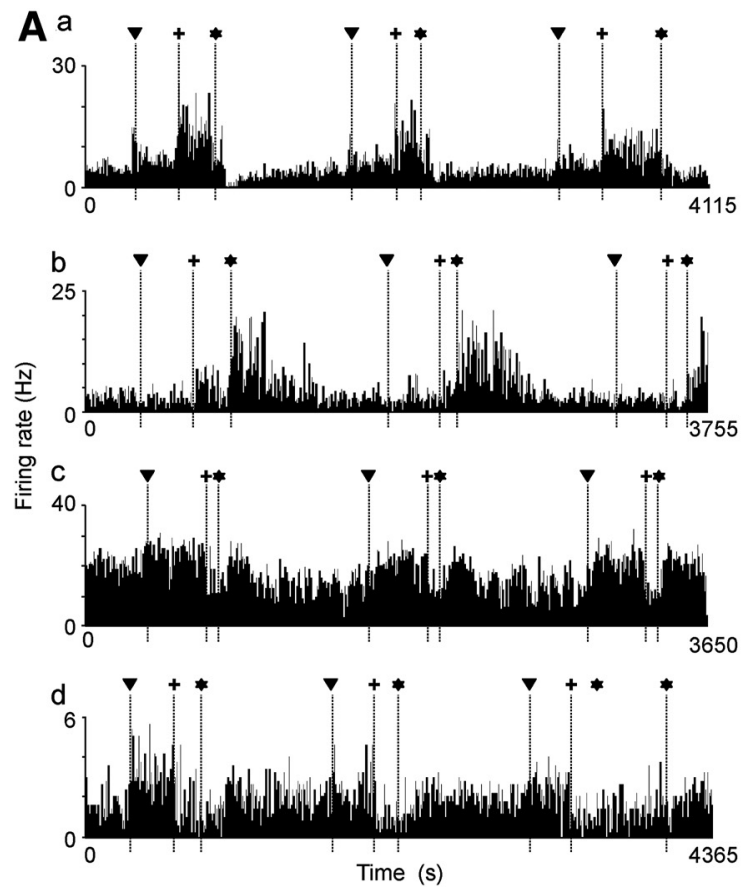

B

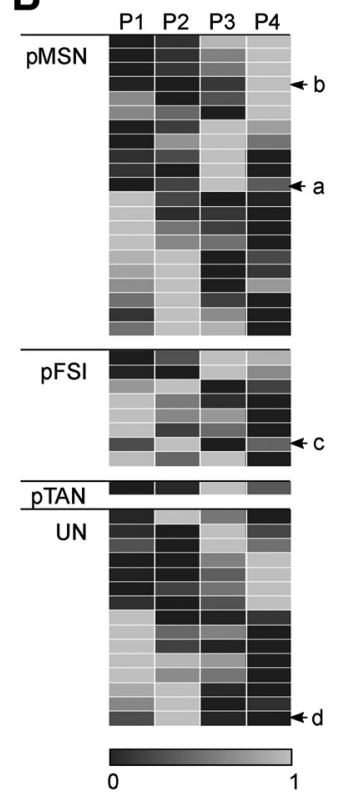

C
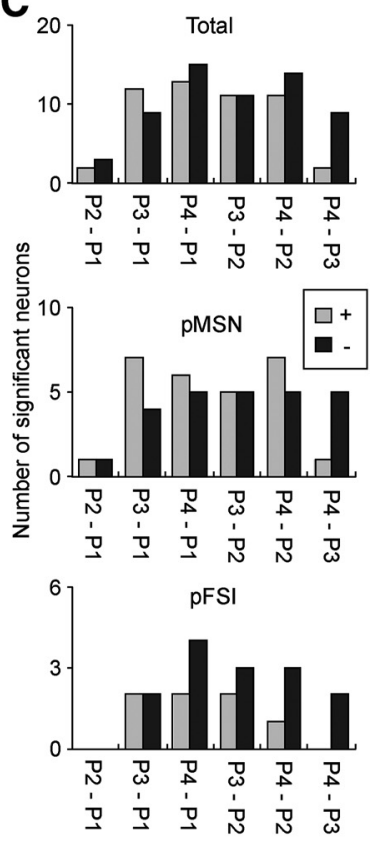

Figure 10. Phase-differential neurons in the NACS. $\boldsymbol{A}$, Four examples of phase-differential neurons ( $\boldsymbol{a}, \boldsymbol{b}, \mathrm{pMSN} ; \boldsymbol{c}, \mathrm{pFSI} ; \boldsymbol{d}$, UN). Histograms indicate instantaneous firing rates throughout a recording experiment. Marks above the histograms correspond to those in Figure 1. B, Normalized firing rates of all phase-differential neurons. P1- 4 corresponds to Phases $1-4$. The normalized mean firing rate in each phase was calculated by the following equation, $\left(F R_{\text {phasex }}-F R_{\text {min }}\right) /\left(F R_{\text {max }}-F R_{\text {min }}\right.$, where $F R_{\text {phasex }^{\prime}} F R_{\text {max }}$ and $F R_{\text {min }}$ represent firing rate in a given phase (Phase $\left.X\right)$ and the maximum and minimum firing rates in all four phases, respectively. C, Number of phase-differential neurons with a significant difference between each pair of phases in all recorded neurons (top), pMSNs (middle), and pFSIs (bottom). P1-4 corresponds to phases 1-4. Gray bars represent the number of neurons with firing rates significantly larger in the former than in the latter phases of the pair. Black bars represent the number of neurons with firing rates significantly smaller in the former than in the latter phases of the pair. Note that the periods during copulation, genital grooming, and sniffing (Figs. 3C, 4C, 5C) were excluded from this analysis.

Table 2. Pearson's correlation coefficients derived from the all NAcS neurons between the two possible phases

\begin{tabular}{lcccc}
\hline & Phase 1 & Phase 2 & Phase 3 & Phase 4 \\
\hline Phase 1 & 1 & & & \\
Phase 2 & 0.24 & 1 & & \\
Phase 3 & -0.42 & -0.33 & 1 & \\
Phase 4 & -0.53 & -0.37 & -0.13 & 1 \\
\hline
\end{tabular}

responsive neurons). Of these, 26 and 14 neurons exhibited excitatory and inhibitory responses, respectively. Examples of pMSN and pFSI responses to sniffing of a female are shown in Figure 7, $A$ and $B$, respectively. The rasters and summed histograms of neuronal activity were aligned with onset and offset of sniffing (Fig. $7 A, B$ ). Underlines under the rasters indicate duration of sniffing. Activities of the pMSN (Fig. 7C) and pFSI (Fig. 7D) significantly increased in Periods $2-3$ and $1-3$ (Bon-
Table 3. Pearson's correlation coefficients derived from the 44 phase-differential neurons between the two possible phases

\begin{tabular}{lcccc}
\hline & Phase 1 & Phase 2 & Phase 3 & Phase 4 \\
\hline Phase 1 & 1 & & & \\
Phase 2 & 0.38 & 1 & & \\
Phase 3 & -0.52 & -0.36 & 1 & \\
Phase 4 & -0.62 & -0.50 & 0.10 & 1 \\
\hline
\end{tabular}

ferroni test after repeated measures one-way ANOVA, $p<$ $0.05)$, respectively. Figure $7 E$ shows response patterns of all the responsive NAcS neurons across the five behavioral periods, and the number of NAcS neurons with significant firing changes in each period is shown in Figure 7F. These results indicate that the NAcS neurons responded to sniffing at various times, and that the response patterns of pMSNs and pFSIs are similar. 

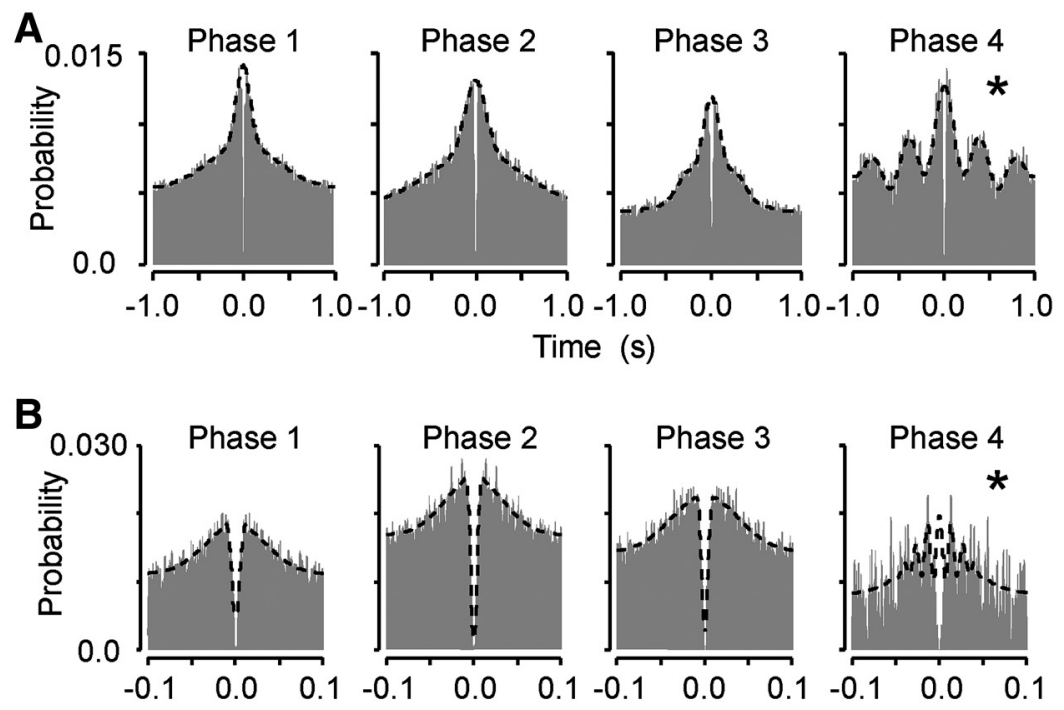

Time (s)

C

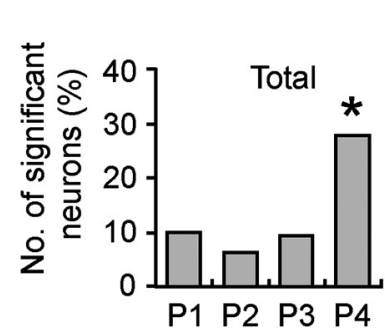

Delta $(1-4 \mathrm{~Hz})$
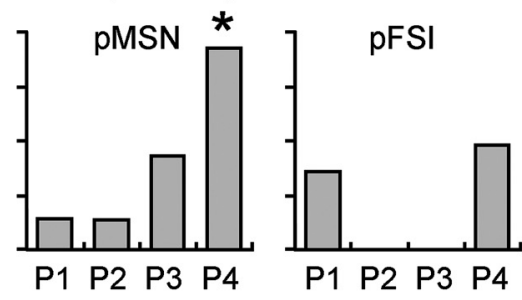

D

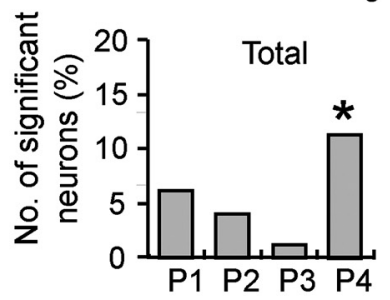

High gamma $(60-80 \mathrm{~Hz})$
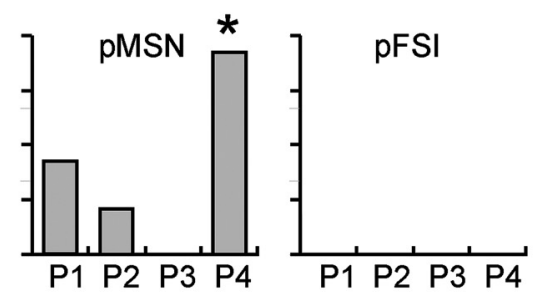

E

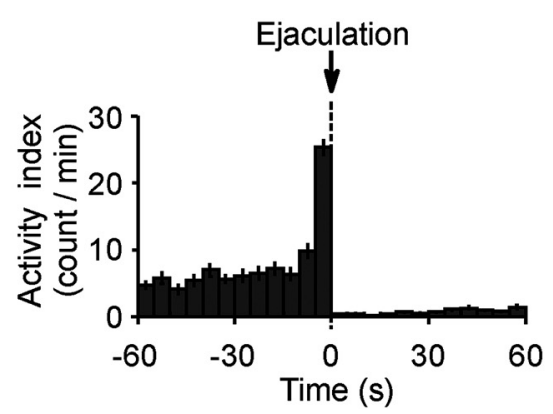

$\mathbf{F}$

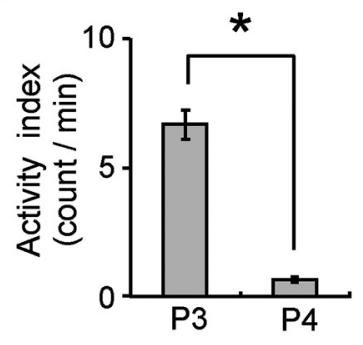

Figure 11. Delta andhigh gamma oscillation in theNACS. $\boldsymbol{A}, \boldsymbol{B}$, Examples of $N A S$ neuronsthatexhibited delta $(\boldsymbol{A})$ orhigh gamma $(\boldsymbol{B})$ oscillation. Histograms indicate auto-correlograms (gray) of each neuron in each phase. The fitted functions (Eq. 1 in the text) are superimposed on the autocorrelograms. * Significantoscillation in delta $(\boldsymbol{A})$ and high gamma $(\boldsymbol{B})$ ranges. $\boldsymbol{C}, \boldsymbol{D}$, Thenumber of $N A$ S Sneurons with significantoscillation in delta $(\boldsymbol{C})$ and high gamma (D) ranges in all recorded neurons (left), pMSNs (middle), and pFSIs (right). P1-P4 represents Phases 1-4. *Significant difference compared with the average number of delta ( $\boldsymbol{C}$ or high gamma $(\boldsymbol{D})$ oscillating neurons (residual analysis, standard residual $>2.0)$. $\boldsymbol{E}$, Perievent histogram of activity index (binsize $=5 \mathrm{~s}$ ). Time 0 indicates the offset of thrusting in ejaculation. Errorbarsindicate SEM.F,Mean activity indexduring Phases 3 and 4 . Error bars indicate SEM. * Significant difference found by paired $t$ test $(p<0.001)$.

Comparison between response patterns of pMSNs and pFSIs to sexual behavior

Ratios of the excitatory and inhibitory neurons in the pMSNs and pFSIs are shown in Figure 8. For intromission + thrust and genital grooming, the ratio of inhibitory neurons was significantly larger in $\mathrm{pFSI}$ than in $\mathrm{pM}$ SNs ( $\chi^{2}$ test, $\left.p<0.05\right)$; such significant differences were not observed for sniffing $\left(\chi^{2}\right.$ test, $p>0.05)$. These results indicate that more pFSIs than pMSNs displayed inhibitory responses to intromission and genital grooming.

Response patterns across the three types of copulatory behavior

Figure 9 shows two examples of NAcS neuron responses to the three different copulatory behaviors (mount + thrust, intromission + thrust, and ejaculation + intromission + thrust), in which rasters and summed histograms of neuronal activities are aligned with onset of thrusting. Activity of the neuron in Figure $9 A$ (pMSN) increased during thrusting, while activity of the neuron in Figure $9 B$ (pFSI) decreased around onset of thrusting. Figure $9, C$ and $D$, shows mean firing rates of the neurons shown in Figure 9, $A$ and $B$, in Period 4 (0.5 $s$ period before onset of thrusting) and Period 5 (thrusting period), respectively. Mean firing rates of the pMSN in Period 5 were significantly higher in intromission + thrust than in mount + thrust (Fig. 9C), while mean firing rates of the pFSI in Period 5 were significantly lower in intromission + thrust than in mount + thrust (Fig. 9D). Table 1 shows the number of neurons with significant differences in their firing rates between intromission + thrust and mount + thrust or between intromission + thrust and ejaculation + intromission + thrust during the prethrust and thrust periods. The numbers of NAcS neurons with differential responses in the thrust periods of mount + thrust and intromission + thrust were larger than chance level for comparisons among all NAcS neurons, pMSNs and pFSIs. However, no such increase was observed in the number of neurons with significant differences between ejaculation + intromission + thrust and intromission + thrust. The results indicate that NAcS neurons responded differentially to mount + thrust and intromission + thrust.

Phase-differential neuronal activity Of 123 NAcS neurons, activity of 44 was significantly different between the four phases (phase-differential neurons). Four examples of phase-differential neurons are shown in Figure $10 \mathrm{~A}$, in which continuous instantaneous activities of the neurons throughout the experiment are shown. Normalized activity patterns of all 44 neurons with significant differences are shown in Figure $10 \mathrm{~B}$. Activity of the neuron shown in Figure $10 \mathrm{Aa}$ increased in Phase 3, activity of the 
neuron shown in Fig. $10 A b$ increased in Phase 4, activity of the neuron shown in Fig.10Ac decreased in Phase 4, and activity of the neuron shown in Fig.10 Ad increased in Phase 2. As shown by these examples, the NAcS neurons displayed various response patterns across the four phases. Figure $10 C$ shows the number of phase pairs with significant differences in neuronal activity. A similar number of neurons displayed significant differences in neuronal activity between all the phase pairs, except the pair of Phases 1 and 2. The results indicate that NAcS neurons are sensitive to phase-specific cues or contexts related to sexual behavior.

Finally, we analyzed whether activity patterns of these 44 neurons allowed discrimination among the four phases. Tables 2 and 3 indicate Pearson's correlation coefficients between the two possible phase pairs using all the NAcS neurons (Table 2) and 44 phase-differential neurons (Table 3). All of the correlation coefficients were $<0.4$ or negative values. These results suggest that these four phases could be discriminated by activity patterns of the NAcS neurons. Furthermore, there were no significant differences in mean firing rates of all 123 NAcS neurons across the four phases $\left(F_{(3,366)}=1.103, p>0.05\right.$; repeated measures one-way ANOVA). This result also held true for the 44 phase-differential neurons as there were no significant differences in mean firing rates of the 44 phase-differential neurons across the four phases $\left(F_{(3,129)}=0.382, p>0.05\right.$; repeated measures one-way ANOVA).

\section{Phase-specific oscillation}

Analysis by auto-correlograms indicated that the NAcS neurons exhibited oscillation in all the frequency ranges of delta $(1-4 \mathrm{~Hz})$, low theta $(4-7 \mathrm{~Hz})$, high theta $(7-12 \mathrm{~Hz}), \alpha(12-20 \mathrm{~Hz})$, low gamma $(40-60 \mathrm{~Hz})$, and high gamma $(60-80 \mathrm{~Hz})$. Of the 123 NAcS neurons, 109 exhibited oscillation in at least one of the four phases in one of six frequency ranges. Figure $11, A$ and $B$, shows two NAcS neurons that oscillated in delta (A) and high gamma (B) frequency ranges in Phase 4. The number of NAcS neurons with delta and/or high gamma oscillations was significantly different among the four phases (Fig. $11 C, D$ ). In both the frequency ranges, the number of oscillated neurons among all the recorded neurons and $\mathrm{pMSN}$ s was significantly larger in Phase 4 than in the other phases $\left(\chi^{2}\right.$ test, $p<0.05$ and residual analysis, standard residual $>2.0$ ). The number of NAcS neurons that oscillated in the other ranges (low theta, high theta, $\alpha$, and low gamma) were not significantly different among the four phases (data not shown).

Increase in oscillations in Phase 4 could be related to behavioral or arousal changes in the postejaculation period (Phase 4). In this phase, rats usually become sexually quiescent and rest for several minutes ( $\mathrm{gmo}, 2007$; Hull and Rodríguez-Manzo, 2009). Consistent with these previous studies, behavioral activity significantly decreased in Phase 4 compared with Phase 3 (paired $t$ test, $p<0.001$ ) (Fig. $11 E, F)$.

\section{Relationship between responsiveness to sexual behaviors and oscillation}

Figure 12 shows a summary of the NAcS neurons that exhibited behavior-related responses (i.e., responses to sexual behaviors and/or phase-differential responses) and the delta oscillation in Phase 4 . Seventy-two percent $(88 / 123)$ of NAcS neurons exhibited behavior-related responses (left column), while 22\% (27/ 123) of NAcS neurons exhibited delta oscillation in Phase 4 (middle column). Furthermore, 74\% (20/27) of the delta oscillating NAcS neurons also exhibited behavior-related firing

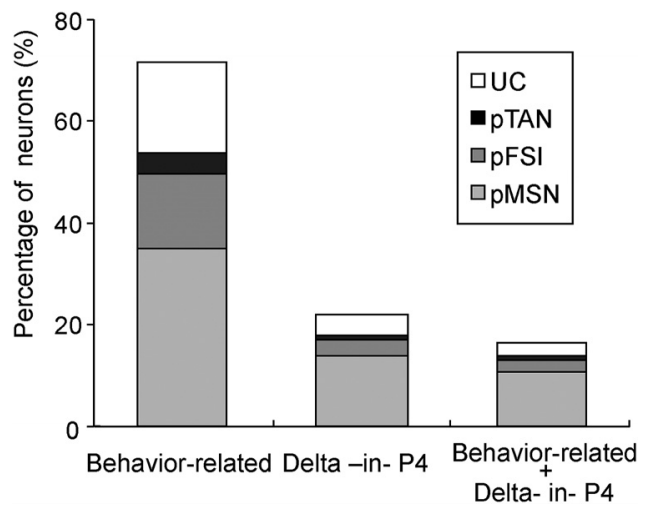

Figure 12. Relationships between behavior-related neurons and delta oscillating neurons in Phase 4. Left column: percentage of behavior-related neurons (behavior-responsive and phasedifferential neurons). Middle column: percentage of neurons that significantly oscillated in the delta range in Phase 4. Right column: percentage of behavior-related neurons that significantly oscillated in the delta range in Phase 4. Gray-colored squares inside the columns indicate the neuronal types shown in the inset.

changes (right column). These results indicate that most delta oscillating neurons exhibited behavior-related activity.

\section{Recording sites of NAcS neurons}

The recording sites of all NAcS neurons (A), pMSNs (B), pFSIs (C), pTANs (D), and UN (E) are shown in Figure 13. All of these neurons were located within the NAcS.

\section{Discussion}

\section{NAcS involvement in performance of sexual behavior}

Previous studies have investigated only four aspects of NAcS activity during sexual behavior: c-fos expression (Robertson et al., 1991; Olivier et al., 2007), tonic dopamine release (Damsma et al., 1992; Fiorino et al., 1997; Lorrain et al., 1999), phasic dopamine release (Robinson et al., 2001, 2002), and local field potentials (LFPs) (Guevara et al., 2008). To our knowledge, no previous studies have investigated the male rat NAcS neuronal activity during sexual behavior. Here, we reported that many NAcS neurons exhibited changed firing rates at various times during sexual behavior. In addition, more pFSIs exhibited inhibitory responses to copulation and genital grooming compared with pMSNs. Finally, neuronal responses to mount + thrust and intromission + thrust were different in some NAcS neurons. These findings represent the first neurophysiological evidence that NAcS neurons encode distinct aspects of sexual behavior, and suggest that the $\mathrm{NAcS}$ is involved in performance of sexual behavior.

\section{NAcS involvement in reward-related functions of sexual behavior}

Tactile stimulation of the penis can be sexually rewarding in rats. In the present study, responses of some NAcS neurons differed between behaviors with and without tactile stimulation of the penis. First, some neurons responded differentially to mount + thrust and intromission + thrust. The penis seems to be more strongly stimulated by rubbing in the vagina in intromission + thrust than in mount + thrust. Furthermore, the intracavernous pressure in the penis is higher during intromission + thrust than during mount + thrust, and penis insertion is presumably facilitated by penile contact with the vaginal orifice ( $\AA$ gmo, 2007). These findings suggest that, before penis insertion into the vagina, the penis is more strongly stimulated by contact with the female body during intromission + thrust than during mount + 


\section{A Total}

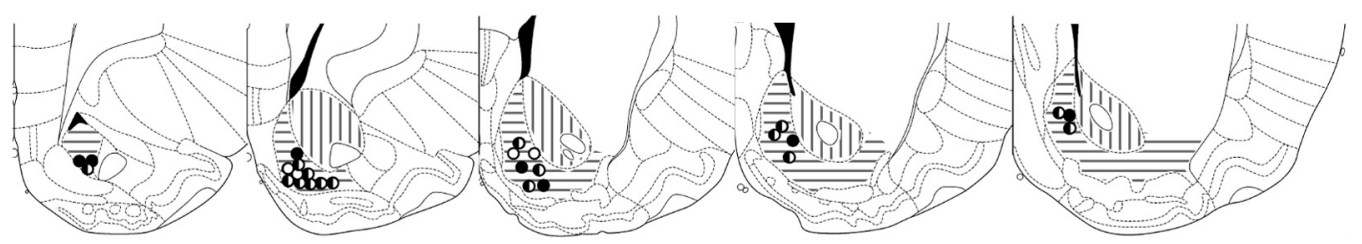

\section{B pMSN}

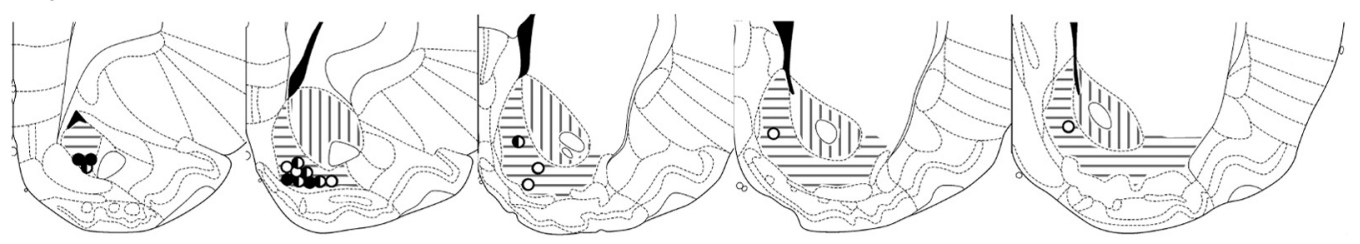

\section{C pFSI}

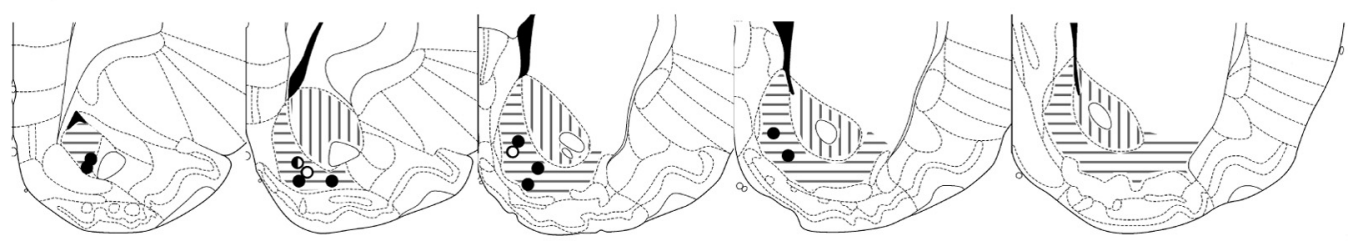

\section{D pTAN}

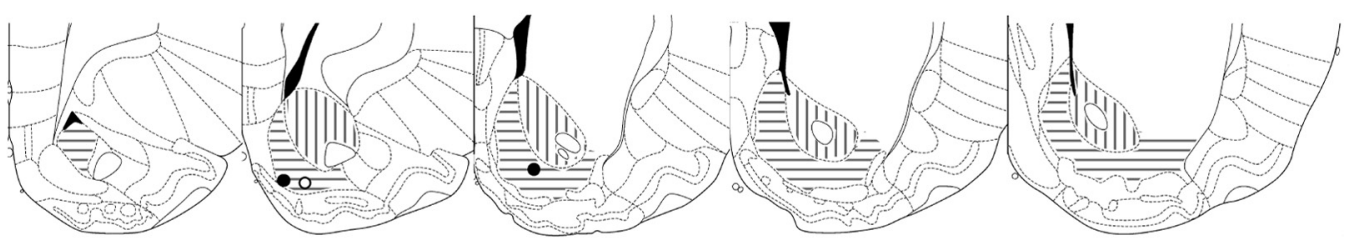

EUN

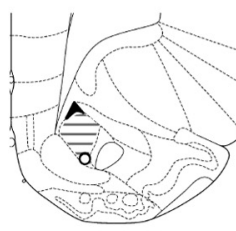

A 3.00

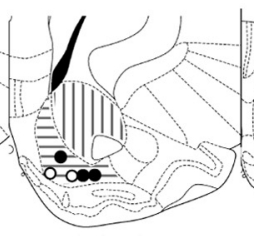

A 2.52

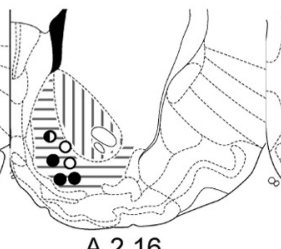

A 2.16

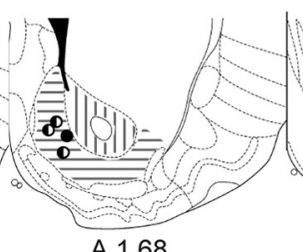

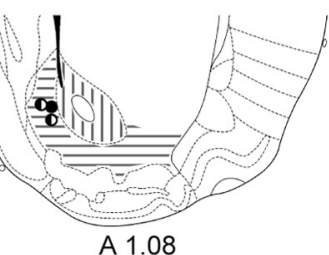

A 1.68

Shell

A 1.08

Behavior-related $\bigcirc$ Behavior -unrelated 1 Both

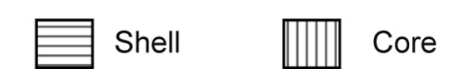

Figure 13. Recording sites of NAcS neurons. Circles indicate histologically identified locations of the tetrode tips, where all NACSs $(\boldsymbol{A})$, pMSNs $(\boldsymbol{B})$, pFSIs $(\boldsymbol{C})$, pTANs $(\boldsymbol{D})$, and UNs $(\boldsymbol{E})$ were recorded. All the neurons were recorded only from the left NAcS. Black circles indicate the locations of the tetrode tips where behavior-related neurons (behavior-responsive and phase-differential neurons) were recorded, open circles indicate locations of the tetrode tips where behavior-unrelated neurons were recorded, half-filled circles indicate locations of the tetrode tips where both the behavior-related and behavior-unrelated neurons were recorded. The area shaded with horizontal and vertical lines indicates the NAcshell and core regions, respectively. Values below each section indicate the distance (in $\mathrm{mm}$ ) anterior from the bregma. Note that, since multiple neurons were usually recorded from each tetrode, the total number of the tetrode tips is smaller than the total number of the neurons.

thrust. These differential responses to mount + thrust and intromission + thrust may reflect the differences in penile stimulation between these two copulatory behaviors. Second, more pFSIs than pMSNs exhibited inhibitory responses to intromission + thrust and genital auto-grooming of the penis, while no such difference between pFSIs and pMSNs was observed with regard to sniffing of females (i.e., appetitive behavior without tactile stimulation of the penis). pFSIs consistently exhibit inhibitory responses during consumption of food rewards (Lansink et al., 2010).

Together, the findings of our study and those of previous studies suggest that the responses to intromission + thrust and genital grooming are involved in reward-related processing. Previous studies have reported that dopamine was also released in the NAc during copulation (Pfaus et al., 1990; Damsma et al., 1992; Fiorino et al., 1997). Dopamine release in the NAc is rewarding (Wise, 2004) and was much higher during copulation than during female presentation without physical contact (Pfaus et al., 1990; Damsma et al., 1992; Fiorino et al., 1997). Most ventral pallidal neurons displayed excitatory responses during reward perception (Tindell et al., 2006), and pallidostriatal neurons selectively send inhibitory projections to parvalbuminimmunoreactive interneurons (FSIs) (Brog et al., 1993; Bevan et al., 1998; Tepper and Plenz, 2006). Furthermore, direct application of dopamine increases the activity of pallidal neurons in vitro (Nakanishi et al., 1985). In addition, some NAc neurons display inhibitory responses to reward through $\mathrm{D}_{2}$ receptors (Tran et al., 
2002). These findings suggest that pFSIs might be inhibited through dopaminergic activity. Genital grooming also seems to be rewarding; electrical stimulation of the dorsal penile nerve and tactile stimulation of the penis increased activity of oxytocin cells in the hypothalamic paraventricular and supraoptic nuclei (Honda et al., 1999; Yanagimoto et al., 1996), which directly or indirectly activate the ventral tegmental area to increase dopamine release in the NAc (Melis et al., 2007, 2009). It is therefore possible that the NAcS is involved in reward-related processing in sexual behavior.

It has been reported that the postejaculatory interval is also rewarding because it can induce conditioned place preference (Pfaus et al., 2001). In this study, the number of high gamma oscillating neurons increased in Phase 4. Gamma oscillation of LFPs in the ventral striatum including the NAcS was associated with food reward perception and neuronal activity in the ventral striatum was phase-locked to the gamma oscillation of the LFPs (Berke, 2009; van der Meer and Redish, 2009; Kalenscher et al., 2010). These findings suggest that the increase of high gamma oscillation in the NAcS after ejaculation may reflect reward perception.

\section{Phase-differential activity in the NAcS}

The present results indicated that activities of 44 neurons were significantly different among the four phases. Moreover, NAcS neuronal activity effectively discriminated among the four phases during sexual behavior. These differential activities could not be ascribed simply to differences in behavior across the four phases because the periods showing the specific behaviors were excluded from the analysis. Furthermore, increase or decrease in sexual arousal cannot explain all the patterns of firing changes across the four phases. Instead, these results strongly suggest that the NAcS is sensitive to different cues or contexts related to sexual behaviors. Consistently, NAc neurons responded more strongly to an odor associated with receptive females than to a neutral odor (West et al., 1992). Furthermore, the NAc receives afferents from the hippocampus (Voorn et al., 2004), which has been suggested to be involved in contextual representation (Smith and Mizumori, 2006).Together, our results, when viewed in context of previous studies, suggest that the NAcS is involved in guiding appropriate sexual behavior in various situations.

\section{Delta oscillation in the postejaculation period (Phase 4)}

After ejaculation, male rats undergo a sexual refractory period, in which the male becomes quiet and loses interest in copulation. In this period, the number of delta oscillating neurons increased. A previous study reported that delta EEG occurred in the NAc during awake immobility, and disappeared with walking (Leung and Yim, 1993). These results suggest that NAcS delta EEGs reflect activity in an inactive idle stage. On the other hand, NAc was suggested to function as a limbic-motor interface, through which drives or emotions processed in the limbic system affect the motor system, based on the afferent inputs to the NAc from the limbic system (Mogenson et al., 1980). Furthermore, most delta oscillating neurons also exhibited behavior-related activity, suggesting that most delta oscillating neurons are involved in behavior-related functions. These findings further suggest that sexual drive cannot be converted into behavioral responses during the postejaculatory period because the NAcS; i.e., the limbicmotor interface, becomes inactive after ejaculation.

It should be noted that, in the present study, the mean firing rates of all NAcS neurons did not decrease in Phase 4 after ejaculation, but the number of delta oscillating neurons significantly increased. Furthermore, the increase in delta oscillation was also observed in pMSNs, which were assumed to be projection neurons in the NAc (Gerfen, 2004). These results suggest that the $\mathrm{NAcS}$ is not simply inactive after ejaculation; it may control behaviors through temporal information, such as delta oscillation.

On the other hand, delta oscillations are typically associated with slow-wave sleep (Steriade et al., 1993) and copulatory behavior with ejaculation increased slow-wave sleep in male rats (Vazquez-Palacios et al., 2002). Furthermore, the NAcS controls the ventrolateral preoptic nucleus that triggers slow-wave sleep (Lazarus et al., 2011). These findings also suggest that delta oscillation in the NAcS might induce drowsiness after ejaculation.

\section{Role of the NAcS in sexual behavior}

The above discussion suggests that there are three possible functions of the NAcS in sexual behavior. First, the NAcS may be involved in reward perception during thrusting and after ejaculation, which induces a drive to copulatory behavior. Second, the NAcS may be involved in execution of appropriate sexual behavior at appropriate times and situations by encoding the cues or contexts related to sexual behavior. Third, the NAcS may be involved in inhibition of copulatory behavior after ejaculation. Consistent with these results, the NAcS receives inputs from the medial amygdala (Brog et al., 1993; Voorn et al., 2004), which processes sexual behavior-related sensory information (e.g., female odor and tactile stimulus to the penis) (Hull and RodríguezManzo, 2009), the medial prefrontal cortex, hippocampus, basolateral amygdala, and ventral tegmental area (Voorn et al., 2004), which are implicated in anticipation, emotion, reward, and context processing (Dalgleish, 2004; Wise, 2004). Both MSNs and FSIs receive these corticolimbic glutamatergic inputs, while FSIs send strong inhibitory GABAergic inputs to MSNs as feedforward inhibition to control the spike timing of MSNs and coordinate oscillation (Tepper and Plenz, 2006). MSNs also send weak GABAergic inputs to nearby MSNs (Tepper and Plenz, 2006). These neural circuits are consistent with our finding that the NAcS neurons displayed excitatory and inhibitory activity changes as well as oscillation during sexual behavior.

Based on these inputs, the NAcS may affect output functions; (1) copulatory behavior through direct or indirect reciprocal connections with the MPOA (Brog et al., 1993), involved in copulation performance (Hull and Rodríguez-Manzo, 2009); (2) motor outputs as a part of the basal ganglia; and (3) emotional/ motivated and autonomic responses via its direct projection to the lateral hypothalamus (Zahm, 1999; Hull and RodríguezManzo, 2009). Our results and previous findings suggest that the NAcS is an important part of a neural circuit that integrates female-related stimuli and genital and motor responses into meaningful mating behavior.

\section{References}

Ågmo A (2007) Functional and dysfunctional sexual behavior: a synthesis of neuroscience and comparative psychology. New York: Academic.

Aragona BJ, Liu Y, Yu YJ, Curtis JT, Detwiler JM, Insel TR, Wang Z (2006) Nucleus accumbens dopamine differentially mediates the formation and maintenance of monogamous pair bonds. Nat Neurosci 9:133-139.

Berke JD (2009) Fast oscillations in cortical-striatal networks switch frequency following rewarding events and stimulant drugs. Eur J Neurosci 30:848-859.

Bevan MD, Booth PA, Eaton SA, Bolam JP (1998) Selective innervation of neostriatal interneurons by a subclass of neuron in the globus pallidus of the rat. J Neurosci 18:9438-9452.

Berke JD (2008) Uncoordinated firing rate changes of striatal fast-spiking interneurons during behavioral task performance. J Neurosci 28:10075-10080. 
Berke JD, Okatan M, Skurski J, Eichenbaum HB (2004) Oscillatory entrainment of striatal neurons in freely moving rats. Neuron 43:883-896.

Bernabé J, Rampin O, Sachs BD, Giuliano F (1999) Intracavernous pressure during erection in rats: an integrative approach based on telematric recording. Am J Physiol 276:441-449.

Brog JS, Salyapongse A, Deutch AY, Zahm DS (1993) The patterns of afferent innervation of the core and shell in the "Accumbens" part of the rat ventral striatum: Immunohistochemical detection of retrogradely transported fluoro-gold. J Comp Neurol 338:255-278.

Cador M, Robbins TW, Everitt BJ (1989) Involvement of the amygdala in stimulus-reward associations: interaction with the ventral striatum. Neuroscience 30:77-86.

Dalgleish T (2004) The emotional brain. Nat Rev Neurosci 5:583-589.

Damsma G, Pfaus JG, Wenkstern D, Phillips AG, Fibiger HC (1992) Sexual behavior increases dopamine transmission in the nucleus accumbens and striatum of male rats: comparison with novelty and locomotion. Behav Neurosci 106:181-191.

Engel AK, König P, Gray CM, Singer W (1990) Stimulus-dependent neuronal oscillations in cat visual cortex: inter-columnar interaction as determined by cross-correlation analysis. Eur J Neurosci 2:588-606.

Everitt BJ (1990) Sexual motivation: a neural and behavioural analysis of the mechanisms underlying appetitive and copulatory responses of male rats. Neurosci Biobehav Rev 14:217-232.

Everitt BJ, Cador M, Robbins TW (1989) Interactions between the amygdala and ventral striatum in stimulus-reward associations: studies using a second-order schedule of sexual reinforcement. Neuroscience 30:63-75.

Everitt BJ, Morris KA, O'Brien A, Robbins TW (1991) The basolateral amygdala-ventral striatal system and conditioned place preference: further evidence of limbic-striatal interactions underlying reward-related processes. Neuroscience 42:1-18.

Fernández-Guasti A, Escalante AL, Ahlenius S, Hillegaart V, Larsson K (1992) Stimulation of 5-HT1A and 5-HT1B receptors in brain regions and its effects on male rat sexual behaviour. Eur J Pharmacol 210:121-129.

Fiorino DF, Coury A, Phillips AG (1997) Dynamic changes in nucleus accumbens dopamine efflux during the Coolidge effect in male rats. J Neurosci 17:4849-4855.

Gerfen C R (2004) Basal ganglia. In: The rat nervous system (Paxinos G, ed), pp 455-508. San Diego: Academic.

Guevara MA, Martinez-Pelayo M, Arteaga Silva M, Bonilla-Jaime H, Hernández-González M (2008) Electrophysiological correlates of the mesoaccumbens system during male rat sexual behaviour. Physiol Behav 95:545-552.

Honda K, Yanagimoto M, Negoro H, Narita K, Murata T, Higuchi T (1999) Excitation of oxytocin cells in the hypothalamic supraoptic nucleus by electrical stimulation of the dorsal penile nerve and tactile stimulation of the penis in the rat. Brain Res Bull 48:309-313.

Hull EM, Rodríguez-Manzo G (2009) Male sexual behavior. In: Hormones, brain and behavior, Vol 1, Ed 2 (Pfaff DW, Arnold AP, Etgen AM, Fahrbach SE, Rubin RT, eds), pp 5-65. San Diego: Academic.

Hull EM, Bitran D, Pehek EA, Warner RK, Band LC, Holmes GM (1986) Dopaminergic control of male sex behavior in rats: effects of an intracerebrally-infused agonist. Brain Res 370:73-81.

Ito R, Robbins TW, Pennartz CM, Everitt BJ (2008) Functional interaction between the hippocampus and nucleus accumbens shell is necessary for the acquisition of appetitive spatial context conditioning. J Neurosci 28:6950-6959.

Johnson RD, Kitchell RL (1987) Mechanoreceptor response to mechanical and thermal stimuli in the glans penis of the dog. J Neurophysiol 57:18131836.

Kalenscher T, Lansink CS, Lankelma JV, Pennartz CM (2010) Rewardassociated gamma oscillations in ventral striatum are regionally differentiated and modulate local firing activity. J Neurophysiol 103:1658-1672.

Kippin TE, Cain SW, Pfaus JG (2003) Estrous odors and sexually conditioned neutral odors activate separate neural pathways in the male rat. Neuroscience 117:971-979.

Kippin TE, Sotiropoulos V, Badih J, Pfaus JG (2004) Opposing roles of the nucleus accumbens and anterior lateral hypothalamic area in the control of sexual behaviour in the male rat. Eur J Neurosci 19:698-704.

König P (1994) A method for the quantification of synchrony and oscillatory properties of neuronal activity. J Neurosci Methods 54:31-37.
Lansink CS, Goltstein PM, Lankelma JV, Pennartz CM (2010) Fast-spiking interneurons of the rat ventral striatum: temporal coordination of activity with principal cells and responsiveness to reward. Eur J Neurosci 32:494-508.

Lazarus M, Shen HY, Cherasse Y, Qu WM, Huang ZL, Bass CE, WinskySommerer R, Semba K, Fredholm BB, Boison D, Hayaishi O, Urade Y, Chen JF (2011) Arousal effect of caffeine depends on adenosine A2A receptors in the shell of the nucleus accumbens. J Neurosci 31: 10067-10075.

Leung LS, Yim CY (1993) Rhythmic delta-frequency activities in the nucleus accumbens of anesthetized and freely moving rats. Can J Physiol Pharmacol 71:311-320.

Liu YC, Sachs BD, Salamone JD (1998) Sexual behavior in male rats after radiofrequency or dopamine-depleting lesions in nucleus accumbens. Pharmacol Biochem Behav 60:585-592.

Lorrain DS, Riolo JV, Matuszewich L, Hull EM (1999) Lateral hypothalamic serotonin inhibits nucleus accumbens dopamine: implications for sexual satiety. J Neurosci 19:7648-7652.

Melis MR, Melis T, Cocco C, Succu S, Sanna F, Pillolla G, Boi A, Ferri GL, Argiolas A (2007) Oxytocin injected into the ventral tegmental area induces penile erection and increases extracellular dopamine in the nucleus accumbens and paraventricular nucleus of the hypothalamus of male rats. Eur J Neurosci 26:1026-1035.

Melis MR, Succu S, Sanna F, Boi A, Argiolas A (2009) Oxytocin injected into the ventral subiculum or the posteromedial cortical nucleus of the amygdala induces penile erection and increases extracellular dopamine levels in the nucleus accumbens of male rats. Eur J Neurosci 30:1349-1357.

Meredith GE (1999) The synaptic framework for chemical signaling in nucleus accumbens. Ann NY Acad Sci 877:140-156.

Mogenson GJ, Jones DL, Yim CY (1980) From motivation to action: functional interface between the limbic system and the motor system. Prog Neurobiol 14:69-97.

Nakanishi H, Hori N, Kastuda N (1985) Neostriatal evoked inhibition and effects of dopamine on globus pallidal neurons in rat slice preparations. Brain Res 358:282-286.

Olivier JD, Jong TR, Jos Dederen PJ, van Oorschot RV, Heeren D, Pattij T, Waldinger MD, Coolen LM, Cools AR, Olivier B, Veening JG (2007) Effects of acute and chronic apomorphine on sex behavior and copulation-induced neural activation in the male rat. Eur J Pharmacol 576:61-76.

Paxinos G, Watson C (2007) The rat brain in stereotaxic coordinates, Ed 6 . New York: Academic.

Pfaus JG, Damsma G, Nomikos GG, Wenkstern DG, Blaha CD, Phillips AG, Fibiger HC (1990) Sexual behavior enhances central dopamine transmission in the male rat. Brain Res 530:345-348.

Pfaus JG, Kippin TE, Centeno S (2001) Conditioning and sexual behavior: a review. Horm Behav 40:291-321.

Robertson GS, Pfaus JG, Atkinson LJ, Matsumura H, Phillips AG, Fibiger HC (1991) Sexual behavior increases c-fos expression in the forebrain of the male rat. Brain Res 564:352-357.

Robinson DL, Phillips PE, Budygin EA, Trafton BJ, Garris PA, Wightman RM (2001) Sub-second changes in accumbal dopamine during sexual behavior in male rats. Neuroreport 12:2549-2552.

Robinson DL, Heien, ML, Wightman RM (2002) Frequency of dopamine concentration transients increases in dorsal and ventral striatum of male rats during introduction of conspecifics. J Neurosci 22:10477-10486.

Schmitzer-Torbert NC, Redish AD (2008) Task-dependent encoding of space and events by striatal neurons is dependent on neural subtype. Neuroscience 153:349-360.

Smith DM, Mizumori SJ (2006) Hippocampal place cells, context, and episodic memory. Hippocampus 16:716-729.

Steriade M, Nuñez A, Amzica F (1993) A novel slow ( $<1 \mathrm{~Hz}$ ) oscillation of neocortical neurons in vivo: depolarizing and hyperpolarizing components. J Neurosci 13:3252-3265.

Tepper JM, Plenz D (2006) Microcircuits in the striatum: striatal cell types and their interaction. In: Microcircuits: the interface between neurons and global brain function (Grillner S, Graybiel AM, eds), pp 127-148. Cambridge: MIT.

Tindell AJ, Smith KS, Peciña S, Berridge KC, Aldridge JW (2006) Ventral pallidum firing codes hedonic reward: when a bad taste turns good. J Neurophysiol 96:2399-2409. 
Tran AH, Tamura R, Uwano T, Kobayashi T, Katsuki M, Matsumoto G, Ono $\mathrm{T}$ (2002) Altered accumbens neural response to prediction of reward associated with place in dopamine D2 receptor knockout mice. Proc Natl Acad Sci U S A 99:8986-8991.

van der Meer MA, Redish AD (2009) Low and high gamma oscillations in rat ventral striatum have distinct relationships to behavior, reward, and spiking activity on a learned spatial decision task. Front Integr Neurosci 3:9

Vazquez-Palacios G, Bonilla-Jaime H, Retana-Marquez S, VelazquezMoctezuma J (2002) Copulatory activity increases slow-wave sleep in the male rat. J Sleep Res 11:237-245.

Voorn P, Vanderschuren LJ, Groenewegen HJ, Robbins TW, Pennartz CM
(2004) Putting a spin on the dorsal-ventral divide of the striatum. Trends Neurosci 27:468-474.

West CH, Clancy AN, Michael RP (1992) Enhanced responses of nucleus accumbens neurons in male rats to novel odors associated with sexually receptive females. Brain Res 585:49-55.

Wise RA (2004) Dopamine, learning and motivation. Nat Rev Neurosci 5:483-494

Yanagimoto M, Honda K, Goto Y, Negoro H (1996) Afferents originating from the dorsal penile nerve excite oxytocin cells in the hypothalamic paraventricular nucleus of the rat. Brain Res 733:292-296.

Zahm DS (1999) Functional-anatomical implications of the nucleus accumbens core and shell subterritories. Ann NY Acad Sci 877:113-128. 